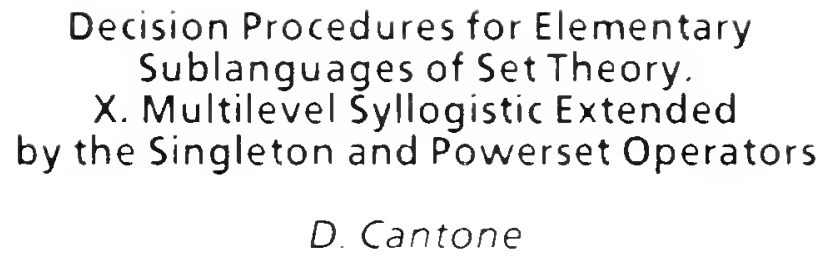

Technical Report 373

May 1988 


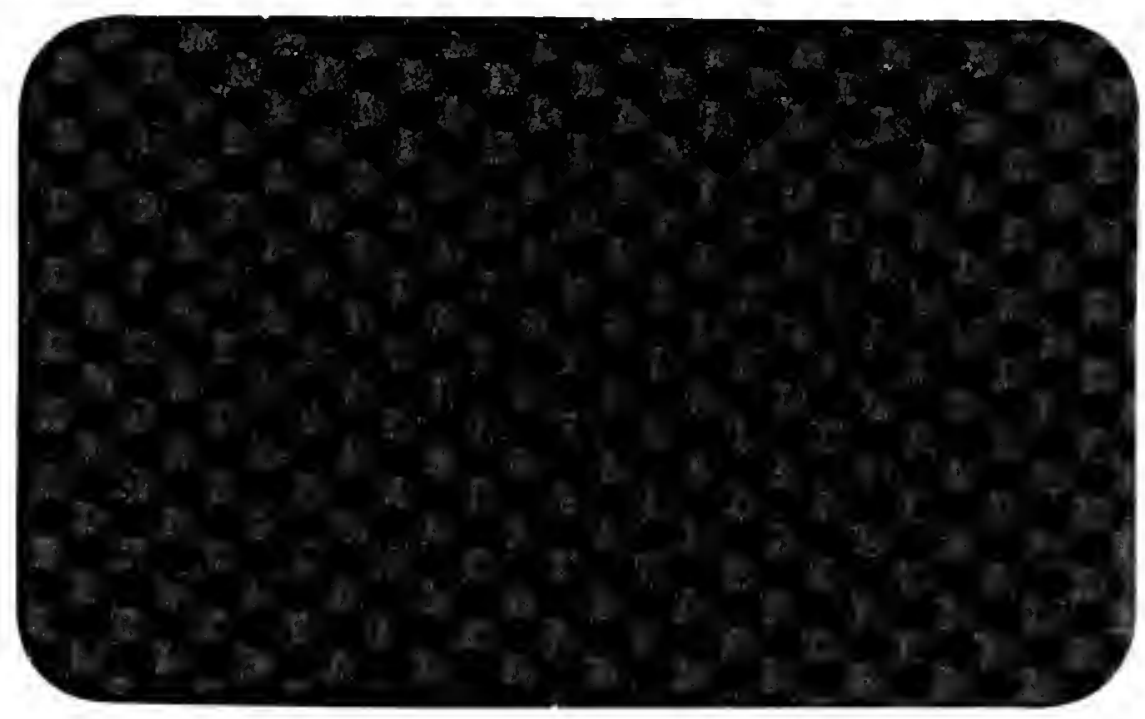


Decision Procedures for Elementary Sublanguages of Set Theory.

$X$. Multilevel Syllogistic Extended

by the Singleton and Powerset Operators

D. Cantone

Technical Report 373

May 1988 



\title{
DECISION PROCEDURES FOR ELEMENTARY SUBLANGUAGES OF SET THEORY. X. MULTILEVEL SYLLOGISTIC EXTENDED BY THE SINGLETON AND POWERSET OPERATORS
}

\author{
D. CANTONE \\ Computer Science Department, \\ Courant Institute of Mathematical Sciences, \\ New York, New York 10012; and \\ Mathematics Department, \\ University of Catania, Catania, Italy
}

\begin{abstract}
The class of unquantified formulae of set theory involving Boolean operators, the powerset and singleton operators, and the equality and membership predicates is shown to have a solvable satisfiability problem.

It is also shown that whenever a formula $\phi$ in the above class is satisfiable there exists a hereditarily finite model of $\phi$, whose rank is bounded by a doubly exponential expression in the number of variables occurring in $\phi$.
\end{abstract}

\section{INTRODUCTION}

Computer verified proofs, even of very elementary mathematical theorems, still require entry of an excessive mass of tedious detail. The same remark applies to the more pragmatic task of program verification. Any hope of making program verification practical will require proof verifiers powerful enough to accept a proof expressed at a level of detail that comes to approximate the mathematical language used in a graduate textbook. Any such verifier will need to incorporate decision procedures for elementary fragments of mathematical theories which 
handle fundamental inference steps much larger than those handled by present verifiers (cf. [Ble3], [BoM], [Con], [KeW], [LuO], [Pas], [Sla], [Tho], and [Wey], for example).

Many efforts have been recently concentrated on inference techniques for various sublanguages of set theory (and, to some extent, analysis) (see $[\mathrm{BrF}]$, [BFOS], [Can], [CFMS], [CFO], [CFOS], [CFS1], [CFS2], [CGO], [COP], [Fer1], [Fer2], [FeO], [FOS1], [FOS2], [Omo], [PaP], [Sch1]).

In [FOS1], the theory MLS (Multi-Level Sillogistic) consisting of formulae built using the boolean connectives (conjunction, disjunction, implication and negation) from set-theoretic atoms of the types

$$
x=y \cup z, x=y \backslash z, x \in y
$$

has been shown to have a solvable satisfiability problem. The satisfiability problem for the extension of MLS obtained by allowing the singleton operator to appear (MLSS theory) was also solved (cf. [FOS1]). In his doctoral dissertation ([Fer1]), Ferro showed that the class of formulae MLS remains still decidable even if at most two occurrences of the powerset operator are allowed; see also the earlier result of [Bre]. This result was subsequently extended in [CFS2] where it is shown that the class of formulae MLSP obtained from MLS br allowing an unrestricted number of occurrences of the powerset also has a solvable satisfiability problem.

This paper describes a decision procedure for a class of set theoretic formulae involving singleton and powerset operators. Specifically, it is shown that the family of unquantified formulae of set theory built up using binary union and intersection, set difference, powerset, singleton, the binary predicates set-membership and equality, and the propositional boolean connectives has a decidable satisfiability problem.

The intended meaning of the language is that in which variables range over (possibly infinite) sets in the standard universe of 'naive' set theory, and the various standard set-theoretic operator and predicate symbols have their standard meanings; hence an interpretation $M$ of a set of sentences $\phi$ of our language is a function which maps every variable $x$ into a set $M x$. If all the sentences of $\phi$ are true under some interpretation of this kind, $\phi$ is said to be satisfiable and each interpretation which satisfies $\mathrm{P}$ is called a model of $\phi$. (Note that all our considerations are easily formalizable in ZFC, and in fact even in weaker set-theoretical systems, since the language with which we work includes only a very few constructs. But we will not belabor this technical point since this paper is concerned with computational rather than foundational questions.) 
The technique used in our solution of the aforementioned satisfiability problem involves an interplay of syntactic and model-theoretic arguments. Firstly, each variable occurring in a normalized conjunction $\phi$ is split into disjoint parts (the places of $\phi$ ), which are essentially syntactic counterparts of the Venn diagram regions of a model of $\phi$. Then it is proved that under the assumption that $\phi$ is satisfiable, $\phi$ must admit a canonical model of rank bounded solely by a function of the size of the conjunction $\phi$ whose satisfiability is to be tested. This is achieved by exhibiting a nondeterministic standardization algorithm consisting of an initialization phase and a stabilization loop. During the initialization phase places are provisionally assigned empty models. The subsequent stabilization phase enlarges the sets corresponding to places in such a way as to ensure that all clauses in $\phi$ are correctly modeled. To prove that such completion can be carried out in an a priori bounded number of steps whenever $\phi$ is satisfiable, an assumed model $M$ of $\phi$ is used as an oracle within a nondeterministic standardization algorithm $w$ hich associates final models with all places of $\phi$.

As a corollary of this construction, it follows that a conjunction $\phi$ is satisfiable if and only if it has a hereditarily finite model of rank bounded by a doubly exponential expression in the number of variables occurring in $\phi$.

\section{PRELIMINARIES}

We denote by MLSSP the propositional closure of atoms of the types

$$
x=y \cup z, x=y \backslash z, x \in y, \quad x=\{y\}, \quad x=\operatorname{pow}(y) .
$$

By using disjunctive normal form and by eliminating inequalities through the axiom of extensionality, the satisfiability problem for MLSSP can be reduced to the satisfiability problem for the subtheory MLSSP' consisting of the conjunctions of literals of the following types,

$$
x=y \cup z, x=y \backslash z, x \in y, x \notin y, x=\{y\}, x=p o w(y) .
$$

For convenience, we will consider a slightly different variant of the satisfiability concept, as described in the following definition.

DEFINITION 2.1 Let $\phi$ be any formula of set theory. An assigniment A of sets to variables occurring in $\phi$ is said to be an injective assignment on $\phi$ if $A x \neq A y$ for all distinct variables $x$ and $y$ in $\phi$.

$\phi$ is said to be injectively satisfiable if there exists an injective assignment which satisfies $\phi$. 
LEMMA 2.2 The satisfiability problem and the injective satisfiability problem for MLSSP' are equivalent.

Proof. It is enough to observe that for every conjunction $\phi$ in MLSSP', $\phi$ is injectively satisfiable if and only if at least one of its variants, obtained by suitably identifying variables in $\phi$, is satisfiable.

It is also helpful to consider the subtheory MLSSP" of MLSSP' consisting of those conjunctions $\phi$ which contain the clauses $q_{0}=q_{0} \backslash q_{0}, p_{0}=\operatorname{pow}\left(q_{0}\right)$, $p_{v}=\operatorname{pow}\left(q_{v}\right), q_{v} \backslash p_{v}=q_{0}$, and $x \in q_{v}$ for all variables $\mathrm{x}$ in $\phi$ distinct from $q_{v}$ and $p_{v}$, where $q_{0}, p_{0}, q_{v}$ and $p_{v}$ are pairwise distinct variables, occurring in $\phi$ only within the above clauses.

Then we have:

LEMMA 2.3 The injective satisfiability problem for MLSSP' is reducible to the injective satisfiability problem for MLSSP".

Proof. It is enough to show that every MLSSP' conjunction can be effectively transformed into an equisatisfiable MLSSP" conjunction. So, let $\phi$ be an MLSSP' conjunction. We let $\tau(\phi)$ denote the conjunction

$\phi \wedge q_{0}=q_{0} \backslash q_{0} \wedge p_{0}=\operatorname{pow}\left(q_{0}\right) \wedge p_{v}=\operatorname{pow}\left(q_{v}\right) \wedge q_{v} \backslash p_{v}=q_{0} \wedge\left(\bigwedge_{\substack{\text { occesv. } \\ \text { ind }}} x \in q_{v}\right)$,

where $q_{0}, p_{0}, q_{v}$ and $p_{v}$ are pairwise distinct variables not occurring in $\phi$. Clearly, if $\tau(\phi)$ is satisfiable, so is $\phi$. Conversely, assume $\phi$ is satisfiable and let $M$ be one of its models. Put $M q_{0}=\emptyset, M p_{0}=\{\emptyset\}$,

$$
M q_{v}=\text { transitive_closure }(\{M x: x \text { occurs in } \phi\}), M p_{v}=\operatorname{pow}\left(M q_{v}\right),
$$

where

$$
\text { transitive_closure }(s)=\bigcap_{\substack{\text { At } \subseteq \text { itanoitive } \\ \text { trats }}} t
$$

and where a set $t$ is said to be transitive if $u \subseteq t$ for all $u \in t$.

It is an easy matter to show that the assignment so extended models correctly $\tau(\phi)$, thus proving that $\phi$ and $\tau(\phi)$ are equisatisfiable.

Next we will introduce some concepts of fundamental relevance in the following sections.

Suppose that a conjunction $\phi$ of MLSSP' is given. 
DEFINITION $2.4 A$ place $\pi$ of $\phi$ is a $0 / 1$-valued function defined on the set of all variables in $\phi$ such that $\pi(x)=\pi(y) \vee \pi(z)$ (resp. $\pi(x)=\pi(y) \wedge \neg \pi(z)$ ) if $x=y \cup z$ (resp. $x=y \backslash z$ ) appears in $\phi$. Moreover, given a variable $x$, the place $\pi$ is said to be a place at $x$ of $\phi$ ) if $\pi(y)=1$ whenever $x \in y$ appears in $\phi$ and $\pi(y)=0$ whenever $x \notin y$ appears in $\phi$.

In the next section we will see that any model of $\phi$ defines naturally a set of places for $\phi$ and places to variables which go a long way toward describing the structure of the model itself.

The following definition introduces a concept of central importance to our purposes.

DEFINITION 2.5 Let $\mathrm{s}$ be a set. Then we put

$$
\operatorname{pow}^{*}(s)=\left\{t: t \subseteq U n(s) \wedge\left(\forall s^{\prime} \in s\right)\left(t \cap s^{\prime} \neq \emptyset\right)\right\}
$$

(where by Un we denote the unary union defined by $U n(s)=\{u: u \in$ $r$, for some $r \in s\}$; cf. [Jec]).

Some immediate properties of the operator pow are listed below.

\section{LEMMA 2.6 (a) pow* ${ }^{*}(\emptyset)=\{\emptyset\}$.}

(b) Let $s_{1}$ and $s_{2}$ be two sets such that $s_{1} \cup s_{2}$ is a collection of pairwise disjoint sets. If pow* $\left(s_{1}\right)=$ pow $^{*}\left(s_{2}\right)$, then $s_{1}=s_{2}$.

(c) Let $\left\{s_{i}: i \in I\right\}$ and $\left\{t_{i}: i \in I\right\}$ be collections of sets such that pow ${ }^{*}\left(\left\{s_{i}:\right.\right.$ $i \in I\}) \subseteq \operatorname{pow}^{*}\left(\left\{t_{i}: i \in I\right\}\right)$.

(d) For each set s,

$$
\operatorname{pow}(U n(s))=U n\left\{\operatorname{pow}^{*}(t): t \in \operatorname{pow}\{s\}\right\}
$$

Proof. (a) is an immediate consequence of the definition of pow*.

Concerning (b), it suffices to observe that if $\operatorname{pow}^{*}\left(s_{1}\right)=\operatorname{pow}^{*}\left(s_{2}\right)$ then $U n\left(s_{1}\right)=U n\left(s_{2}\right)$. The latter equality combined with the disjointness hypothesis implies $s_{1}=s_{2}$.

Concerning (c), let $\left\{s_{i}: i \in I\right\}$ and $\left\{t_{i}: i \in I\right\}$ be such that $s_{i} \subseteq t_{i}$ for all $i \in I$. Plainly, $U n\left(\left\{s_{i}: i \in I\right\}\right) \subseteq U n\left(\left\{t_{i}: i \in I\right\}\right)$. Moreover, if $u \cap s_{i} \neq \emptyset$ for all $i \in I$, then a fortiori $u \cap t_{i} \neq \emptyset$, for all $i \in I$. Hence $\operatorname{pow}^{*}\left(\left\{s_{i}: i \in I\right\}\right) \subseteq \operatorname{pow}^{*}\left(\left\{t_{i}: i \in I\right\}\right)$.

Finally, as regards (d), let $u \in \operatorname{pow}\left(U_{n}(s)\right)$, and let $t_{\mathrm{u}}=\{t \in s: t \cap$ $u \neq \emptyset\}$. Plainly $u \in$ pow $\left(t_{u}\right)$ and $t_{u} \in \operatorname{pow}(s)$, which prove pow $(\operatorname{Un}(s)) \subseteq$ 
$U n\left\{\operatorname{pow}^{*}(t): t \in \operatorname{pow}(s)\right\}$. Also, as pow* $(t) \subseteq \operatorname{pow}(U n(t)) \subseteq$ pow $(U n(s))$, for all $t \in$ pow $(s)$, the converse inclusion follows at once, thereby proving (d), and in turn concluding the proof of the lemma.

Remark. Throughout this paper, for any given mapping $f: X \rightarrow Y$, we denote by $\bar{f}$ the mapping from pow $(X)$ into pow $(Y)$ defined by

$$
\bar{f}(A)=f[A], \text { for all } A \in \operatorname{pow}(X) .
$$

The following properties hold.

LEMMA 2.7 Let $f$ be a 1-1 it function. Then,

(a) $\bar{f}$ is $1-1$.

(b) If $s \subseteq p o w(\operatorname{dom}(f))$, then

(b.1) $\operatorname{pow}^{*}(s) \subseteq \operatorname{dom}(\bar{f})$, and

(b.2) $\bar{f}\left[\right.$ pow $\left.^{*}(s)\right]=\operatorname{pow}^{*}(\bar{f}[s])$.

Proof. Concerning (a), let $s_{1}$ and $s_{2}$ be two distinct subsets of $\operatorname{dom}(f)$, and let $u \in\left(s_{1} \backslash s_{2}\right) \cup\left(s_{2} \backslash s_{1}\right)$. Then $f(u) \in f\left[\left(s_{1} \backslash s_{2}\right) \cup\left(s_{2} \backslash s_{1}\right)\right]=\left(f\left[s_{1}\right] \backslash f\left[s_{2}\right]\right) \cup$ $\left(f\left[s_{2}\right] \backslash f\left[s_{1}\right]\right)$, which implies $f\left[s_{1}\right] \neq f\left[s_{2}\right]$, i.e. $\bar{f}$ is injective.

As regards (b), let $s \subseteq$ pow $(\operatorname{dom}(f))$. Then for each $t \in$ pow $^{*}(s), t \subseteq$ $U n(s) \subseteq \operatorname{dom}(f)$, i.e. $t \in \operatorname{dom}(\bar{f})$. Therefore pow* $(s) \subseteq \operatorname{dom}(\bar{f})$, which proves (b.1). To prove (b.2), let $t \in \operatorname{pow}^{*}(s)$. As $t \subseteq U n(s)$, we have $\bar{f}(t)=f[t] \subseteq$ $f[U n(s)]=U n(\bar{f}[s])$. Moreover, $t \cap s^{\prime} \neq \emptyset$ for each $s^{\prime} \in s$. Hence $\bar{f}(t) \cap u \neq \bar{\emptyset}$, for all $u \in \bar{f}[s]$. Thus $\bar{f}(t) \in$ pow $^{*}(\bar{f}[s])$, which proves $\bar{f}\left[\right.$ pow $\left.^{*}(s)\right] \subseteq$ pow $^{*}(\bar{f}[s])$. Conversely, let $w \in \operatorname{pow}^{*}(\bar{f}[s])$. Then $w \in U n(\bar{f}[s]) \subseteq f[U n(s)]$. Put $u=$ $f^{-1}[w]$. Plainly $u \subseteq U n(s)$. Moreover, for all $s^{\prime} \in s, w \cap f\left[s^{\prime}\right]=w \cap \bar{f}\left(s^{\prime}\right) \neq \emptyset$, so that $u \cap s^{\prime}=f^{-1}[w] \cap f^{-1} f\left[s^{\prime}\right]=f^{-1}\left[w \cap f\left[s^{\prime}\right]\right] \neq \emptyset$. Hence $u \in$ pow $w^{*}(s)$, and since $w=f f^{-1}[w]=f[u]=\bar{f}(u)$, we obtain $w \in \bar{f}\left[\right.$ pow $\left.^{*}(s)\right]$ which in turn implies pow* $(\bar{f}[s]) \subseteq \bar{f}\left[\right.$ pow $\left.^{*}(s)\right]$. This concludes the proof of (b.2), so that the lemma is completely proved.

We close this section by defining the von Neumann hierarchy of sets and by introducing the notion of rank of a set. Specifically, for all ordinals $\alpha$, put inductively

$$
\begin{aligned}
\mathcal{V}_{0} & =\emptyset \\
\mathcal{V}_{\alpha+1} & =\operatorname{pow}\left(\mathcal{V}_{\alpha}\right) \\
\mathcal{V}_{\alpha} & =\bigcup_{\beta<\alpha} \mathcal{V}_{\beta}, \text { if } \alpha \text { is a limit ordinal. }
\end{aligned}
$$


Finally, the von Neumann universe of sets is

$$
\mathcal{V}=\bigcup_{\alpha \in O_{r d}} \mathcal{V}_{\alpha} .
$$

It follows from the axiom of regularity that every set belongs to some set $\mathcal{V}_{\alpha}$ (see for example [Jec]). Therefore for every set $s$ we mas define the rank of $s$ by putting

$$
\operatorname{rank}(s)=\text { least } \alpha \text { such that } s \in \mathcal{V}_{a+1} \text {. }
$$

Some of the most useful properties of the rank function are:

(i) for every ordinal number $\alpha, \operatorname{rank}(\alpha)=\alpha$;

(ii) $\operatorname{rank}\left(\left\{s_{0}, s_{1}, \ldots, s_{n}\right\}\right)=\max _{i \in\{0,1, \ldots, n\}} \operatorname{rank}\left(s_{i}\right)+1$;

(iii) $\operatorname{rank}(s)=\bigcup_{\epsilon_{s}}(\operatorname{rank}(r)+1)=\sup _{r \in s}(\operatorname{rank}(r)+1)$;

(iv) if $r \in s$, then $\operatorname{rank}(r)<\operatorname{rank}(s)$;

(v) $\operatorname{rank}(s \cup t)=\max \{\operatorname{rank}(s), \operatorname{rank}(t)\}$;

(vi) $\operatorname{rank}\left(\bigcup_{i \in I} s_{i}\right)=\bigcup_{i \in I} \operatorname{rank}\left(s_{i}\right)$;

(vii) $\mathcal{V}_{\alpha}=\{s: \operatorname{rank}(s)<\alpha\}$.

The members of $\mathcal{V}_{\omega}$, i.e. the sets having finite rank, are called hereditarily finite sets.

\section{THE MAIN RESULT}

In the preceding section we have shown that the satisfiability problem for the class of formulae MLSSP can be reduced to the injective satisfiability problem for the narrower class MLSSP". This section solves this latter problem.

The following theorem gives decidable conditions for a conjunction $\phi$ in MLSSP" to be injectively satisfiable.

THEOREM 3.1 Let $\phi$ it be a conjunction of literals each of which it has one of the following types,

$$
\begin{array}{ll}
(=) & x=y \cup z, x=y \backslash z \\
(\in, \notin) & x \in y, x \notin y \\
(\{\cdot\}) & x=\{y\} \\
(\text { pow }) & x=\operatorname{pow}(y) .
\end{array}
$$


Assume also that $\phi$ contains the clauses

$$
q_{0}=q_{0} \backslash q_{0}, \quad p_{0}=\operatorname{pow}\left(q_{0}\right), \quad p_{v}=\operatorname{pow}\left(q_{v}\right), \quad q_{v} \backslash p_{v}=q_{0}, x \in q_{v},
$$

for all variables $\mathrm{x}$ in $\phi$ distinct from $q_{v}$ and $p_{v}$, where $q_{0}, p_{0}, q_{v}$, and $p_{v}$ occur in $\phi$ only within the clauses (1). Also, let $p_{0}=p o w\left(q_{0}\right), p_{1}=p o w\left(q_{1}\right), \ldots, p_{v-1}=$ pow $\left(q_{v-1}\right), p_{v}=\operatorname{pow}\left(q_{v}\right)$ be all powerset clauses in $\phi$, and let $\mathrm{V}$ be the collection of distinct variables occurring in $\phi$. Then $\phi$ is injectively satisfiable, i.e. satisfiable by a model which maps distinct variables into distinct sets, if and only if there exist

(i) a set $\mathrm{II}=\left\{\pi_{1}, \ldots, \pi_{n}\right\}$ of places of $\phi$;

(ii) a mapping $x \mapsto \pi^{x}$ from $V \backslash\left\{p_{v}\right\}$ into II;

(iii) a mapping $x \mapsto \bar{\pi}$ from $\Pi$ into the von Neumann universe of sets such that

(C1) no two distinct variables in $\phi$ are II-equivalent (i.e. for every $x, y$ in $\phi$, with $x \neq y$, there is a place $\pi \in \Pi$ such that $\pi(x) \neq \pi(y)$ );

(C2) for each variable $x$ in $V \backslash\left\{p_{v}\right\}$ the place $\pi^{x}$ is at the variable $x$;

(C3.a) $\bar{\pi} \neq \emptyset$, for all places $\pi \in \Pi$;

(C3.b) $\bar{\alpha} \cap \bar{\beta}=\emptyset$, for all places $\alpha, \beta \in \Pi$, with $\alpha \neq \beta$;

(C3.c) $\bigcup_{x(x)=1} \bar{\pi} \in \overline{\pi^{x}}$, for all variables $x$ in $V \backslash\left\{p_{v}\right\}$;

(C3.d) $\bigcup_{\alpha(p)=1} \bar{\alpha}=\operatorname{pow}\left(\cup_{\beta(q)=1} \bar{\beta}\right)$, for all powerset clauses $p=p o w(q)$ in $\phi$;

(C3.e) $\bigcup_{\alpha(x)=1} \bar{\alpha}=\left\{\bigcup_{\beta(y)=1} \bar{\beta}\right\}$, for all singleton clauses $x=\{y\}$ in $\phi$;

(C4) it must be possible to produce the mapping $\pi \mapsto \bar{\pi}$ in (iii) by an execution of the following nondeterministic association algorithm, in which Step 2 is executed at most $(\rho-2)(n-1) 2^{n-2}+3 \cdot 2^{n-1}-2$ times, where $n=|\Pi|$, and $\rho$ is any natural number greater than 1 and such that $2^{\rho-1}>\rho(n-1)+1$.

\section{ASSOCIATION ALGORITHM}

Step 1. Put

$$
\bar{\pi} \leftarrow \emptyset,
$$

for all places $\pi \in \mathrm{II}$. 
Step 2. Pick a set $\left\{\alpha_{1}, \ldots, \alpha_{\ell}\right\} \subseteq \mathrm{II}$ and choose sets $\Delta_{\pi}$, with $\pi \in \mathrm{II}$, such that

$$
\bigcup_{\pi \in \Pi} \Delta_{\pi} \subseteq \text { pow }^{*}\left(\left\{\overline{\alpha_{1}}, \ldots, \overline{\alpha_{\ell}}\right\}\right) .
$$

Enlarge each set $\bar{\pi}$ by putting

$$
\bar{\pi} \leftarrow \bar{\pi} \cup \Delta_{\pi}
$$

Step 3. Stop or to go Step 2.

Proof. Sufficiency. Assume, first, that there exist II, $x \mapsto \pi^{x}, \pi \mapsto \bar{x}$ as in (i), (ii), and (iii) and such that conditions (C1)-(C4) are all satisfied. Then we will prove that the assignment $M^{*}$ defined on the variables of $\phi$ by

$$
M^{*} x=\bigcup_{\pi(x)=1} \bar{\pi}
$$

is injective and satisfies all the conjuncts in $\phi$.

Let $x, y$ be variables occurring in $\phi$, and assume that $M^{*} x=M^{*} y$. We will show that $x \equiv y$. Indeed, since $\bigcup_{\pi(x)=1} \bar{\pi}=\bigcup_{\pi(y)=1} \bar{\pi}$, conditions (C3.a) and (C3.b) imply that $x$ and $y$ are $\Pi$-equivalent, which by (C1) gives $x \equiv y$.

Next we prove that $M^{*}$ satisfies each conjunct in $\phi$. Let $x=y \cup z$ (resp. $x=y \backslash z$ ) occur in $\phi$. In view of (??) and conditions (C3.a) and (C3.b), in order to prove that $M^{*} x=M^{*} y \cup M^{*} z$ (resp. $M^{*} x=M^{*} y \backslash M^{*} z$ ) it suffices to show that $\pi(x)=\pi(y) \vee \pi(z)$ (resp. $\pi(x)=\pi(y) \wedge \neg \pi(z)$ ), for all $\pi \in \Pi$. But this follows immediately from Definition 2.4.

Having proved that $M^{*}$ models correctly all literals in $\phi$ of type (=), we show that membership relations are also satisfied. Let the clause $x \in y$ belong to $\phi$. By $(\mathrm{C} 2), \pi^{x}(y)=1$. Hence (??) and (C3.c) imply $M^{*} x=\bigcup_{\pi(x)=1} \bar{\pi} \in$ $\overline{\pi^{x}} \subseteq \bigcup_{\pi(y)=1} \bar{\pi}=M^{*} y$, i.e. $M^{*} x \in M^{*} y$.

On the other hand, if $x \notin y$ occurs in $\phi$, then $\pi^{x}(y)=0$, so that by (C3.b) we have $\overline{\pi^{x}} \cap M^{*} y \neq \emptyset$, and therefore (C3.c) implies $M^{*} x \notin M^{*} y$.

Finally, in view of (??), conditions (C3.d) and (C3.e) respectively imply that powerset and singleton clauses in $\phi$ are satisfied by $M^{*}$, thereby completing the proof that $M^{*}$ injectively satisfies $\phi$.

Necessity. Next assume that there exists an injective model $M$ of $\phi$.

The following lemma lists some properties of the model $M$ which are immediate consequences of clauses (1). 
LEMMA 3.2 (1) $M q_{0}=\emptyset, M p_{0}=\{\emptyset\}$;

(2) $M q_{v}$ and $M p_{v}$ are transitive sets;

(3) $M x \subseteq M p_{v}$, for all $x \in V$;

(4) $M y \in M p_{v}$ and $M y \subseteq M q_{v}$, for all $y \in V \backslash\left\{p_{v}\right\}$;

(5) $M z \in M q_{v}$, for all $z \in V \backslash\left\{q_{v}, p_{v}\right\}$.

Next we proceed to the construction of $\Pi, x \mapsto \pi^{x}$, and $\pi \mapsto \bar{\pi}$ as from (i)-(iii).

Let $\sigma_{1}, \sigma_{2}, \ldots, \sigma_{n}$ be the nonempty disjoint parts of the Venn diagram of the collection of sets $\left\{M y: y \in V \backslash\left\{p_{v}\right\}\right\}$ in the universe

$$
\mathcal{U}=\left(\bigcup_{y \in V} M y\right) \cup \bigcup_{y \in V \backslash\left\{p_{v}\right\}}\{M y\}=M p_{v} .
$$

Then for every set $\sigma_{i}$ we define

$$
\pi_{i}(y)=\left\{\begin{array}{ll}
0 & \text { if } \sigma_{i} \cap M y=\emptyset \\
1 & \text { if } \sigma_{i} \subseteq M y
\end{array},\right.
$$

where $y$ ranges over $V$, and we put $\Pi=\left\{\pi_{1}, \ldots, \pi_{n}\right\}$. Furthermore, given a variable $y$ in $V \backslash\left\{p_{v}\right\}$, we put $\pi^{y}=\pi_{i y}$, where $M y \in \sigma_{i,}$, for some $i_{y} \in$ $\{1,2, \ldots, n\}$.

Remark. For each $\pi \in \Pi$, we designate by $\sigma^{\pi}$ the region of the Venn diagram relative to $M$ and $\phi$ which induces the place $\pi$.

It is an easy matter to verify that the $\pi^{\prime} s$ are places of $\phi$ and that for each $y$ in $V \backslash\left\{p_{v}\right\}$, the place $\pi^{y}$ is at the variable $y$. Thus (i), (ii), and (C2) hold.

Next we prove that $(\mathrm{C} 1)$ holds too. Let $x, y$ be two distinct variables occurring in $\phi$. Since $M x \neq M y$, there exists $s \in(M x \backslash M y) \cup(M y \backslash M x)$, so that $s \in \mathcal{U}$ (where $\mathcal{U}$ is the universe). Let $\sigma_{i_{0}}$ be the region of the Venn diagram which contains the set $s$. Whence $\pi_{i_{0}}(x) \neq \pi_{i_{0}}(y)$, showing that $x$ and $y$ are not $\Pi$-equivalent.

To complete the proof of the necessity of conditions (C1)-(C4) we need to exhibit an instantiation of the Association Algorithm which produces the sets $\bar{\pi}$ in at most

$$
(\rho-2)(n-1) 2^{n-2}+3 \cdot 2^{n-1}-2
$$

executions of Step 2 and such that (C3.a)-(C3.e) are satisfied. This is accomplished by using the given model $M$ as an 'oracle' which permits us to 'extract' a transformed canonical model of $\phi$. Such canonical model will have rank bounded by a doubly exponential expression in the size of $V$. 
The following lemma expresses some useful facts about the regions of the Venn diagram relative to $M$ and $\phi$ and their corresponding places.

LEMMA 3.3 (1) $\sigma^{\pi^{90}}=\{0\}$ and $\pi^{90}$ is the unique place $\pi \in$ II such that $\pi(p)=1$ for all powerset clauses $p=$ pow $(q)$ in $\phi$.

$\sigma^{\pi_{v}}=M p_{v} \backslash M q_{v}$. Hence $\pi^{q_{v}}\left(p_{v}\right)=1$ and $\pi^{q_{v}}\left(q_{v}\right)=0$.

(3) $\pi\left(q_{v}\right)=1$, for each $\pi \in \Pi \backslash\left(\pi^{q v}\right)$.

(4) $n=|\Pi| \leq 2^{|V|-1}+1$.

(5) If $x=\{y\}$ occurs in $\phi$, then $\pi^{y}$ is the unique place $\pi \in \Pi$ such that $\pi(x)=1$.

Proof. As $=M q_{0} \in \sigma^{\pi^{80}} \cap M p_{0}$, then $\sigma^{\pi^{80}}=M p_{0}=\{\theta\}$, which implies $\sigma^{\pi^{90}}=\{\emptyset\}$. Therefore $\sigma^{\pi^{90}} \subseteq$ pow $(s)$ for all sets $s$ and in particular $\sigma^{\pi^{90}} \subseteq M_{p}$, i.e. $\pi^{90}(p)=1$, for all powerset clauses $p=\operatorname{pow}(q)$ in $\phi$. The uniqueness of $\pi^{90}$ follows plainly since $q_{0}=q_{0} \backslash q_{0} \wedge p_{0}=$ pow $\left(q_{0}\right)$ occurs in $\phi$. Thus $(1)$ is proved.

Concerning (2), since $M q_{v} \in \sigma^{\pi^{q_{v}}}$, we have $\sigma^{\pi^{q_{v}}} \subseteq M p_{v} \backslash M q_{v}$. If $\sigma^{\pi} \subseteq$ $M p_{v} \backslash M q_{v}$ for some $\pi \neq \pi^{q v}$, then there would exist a variable $\mathrm{y}$ in $\phi$ such that

$$
\sigma^{\pi_{0}} \cap M y=\emptyset \text { and } \sigma^{\pi_{1}} \subseteq M y
$$

where $\left\{\pi_{0}, \pi_{1}\right\}=\left\{\pi, \pi^{q *}\right\}$. Hence from Lemma $3.2(3)$ it would follow $y \neq p_{v}$ which by (4) of the same lemma would imply $M y \subseteq M q_{v}$. This inclusion in turn would gire $\sigma^{\pi_{1}} \subseteq M q_{v}$, a contradiction. Therefore $\sigma^{\pi^{q^{*}}}=M p_{v} \backslash M q_{v}$, and (2) is proved.

Next, to prove (3) we consider $\pi \in \Pi \backslash\left\{\pi^{q \bullet}\right\}$. Lemma $3.2(3)$ implies $\sigma^{\pi} \subseteq$ $M p_{v} \backslash \sigma^{\pi^{q v}}=M q_{v}$, which yields $\pi\left(q_{v}\right)=1$.

Concerning (4) it suffices to note that by (3) $\left|\left\{\pi \in \Pi: \pi\left(q_{v}\right)=1\right\}\right| \leq 2^{|v|-1}$.

Finally, to prove (5) we observe that $M y \in \sigma^{\pi^{y}} \subseteq M x$, so that $\sigma^{\pi^{y}}=$ $\{M y\}=M x$, showing that $\pi^{y}$ is the unique place $\pi \in \Pi$ such that $\pi(x)=1$. Thus the proof of the lemma is completed.

Next we introduce the concepts of $\mathrm{P}$-nodes and their P-targets, which will play a central role in the proof of the necessity of conditions (C4) and (C3).

Let pow" $\left(\left\{\sigma^{\alpha_{1}}, \ldots, \sigma^{\alpha_{\ell}}\right\}\right) \cap \sigma^{\beta} \neq \emptyset$, for some regions $\sigma^{\alpha_{1}}, \ldots, \sigma^{\alpha_{\ell}}, \sigma^{\beta}$ of the Venn diagram relative to $M$ and $\phi$. Then there exists $s \in p o w^{\bullet}\left(\left\{\sigma^{\alpha_{1}}, \ldots, \sigma^{\alpha_{\ell}}\right\}\right) \cap$ $\sigma^{\beta}$, and we can write $s=\bigcup_{i=1}^{\ell}\left(s \cap \sigma^{\alpha_{i}}\right)$, where all the sets $s \cap \sigma^{\alpha_{i}}$ appearing in the union are nonempty. For every powerset clause $p=p o w(q)$ in $\phi$ such that $\beta(p)=1$, we have $\sigma^{\beta} \subseteq M p$ and consequently $s \in$ pow $(M q)$. Thus $s \cap \sigma^{\alpha_{i}} \subseteq M q$ and hence $\sigma_{\alpha_{1}} \subseteq M q \subseteq M q_{v}$ for all $i=1, \ldots, \ell$, implying that $\alpha_{i}(q)=1$ (and 
$\left.\alpha_{i}\left(q_{v}\right)=1\right)$ for all $i=1, \ldots, \ell$. In the same way it can be shown that for a given powerset clause $p=\operatorname{pow}(q)$ in $\phi$, if $\alpha_{i}(q)=1, i=1, \ldots, \ell$, then $\beta(p)=1$. These semantic considerations suggest the following definition, which is purely syntactic.

DEFINITION 3.4 (a) Any subset of $\Pi \backslash\left\{\pi^{q *}\right\}$ is called a P-node.

(b) Let A be a P-node. A place $\beta$ is called a P-target (or simply a target) of A if for every powerset clause $p=p o w(q)$ in $\phi$ we have

$$
\beta(p)=1 \text { if and only if } \alpha(q)=1 \text {, for all } \alpha \in A \text {. }
$$

Remark. In the following for each $P$-node $A$ we will write $T(A)$ to denote the set of P-targets of $\mathrm{A}$, so that $\mathrm{T}$ maps the set pow( $\left.\Pi \backslash\left\{\pi^{q *}\right\}\right)$ into pow( $\left.\Pi\right)$.

The discussion preceding Definition 3.4 is now restated in terms of the new concepts just introduced.

LEMMA 3.5 Let $\operatorname{pow}^{*}\left(\left\{\sigma^{\alpha_{1}}, \ldots, \sigma^{\alpha_{\ell}}\right\}\right) \cap \sigma^{\beta} \neq 0$, it for some places $\alpha_{1}, \ldots, \alpha_{\ell}, \beta$ of $\phi$. Then $\left\{\alpha_{1}, \ldots, \alpha_{\ell}\right\}$ is a $\mathrm{P}$-node and $\beta$ is a torget of $\left\{\alpha_{1}, \ldots, \alpha_{\ell}\right\}$, i.e. $\beta \in T\left(\left\{\alpha_{1}, \ldots, \alpha_{\ell}\right\}\right)$.

The following lemma states other useful facts about $\mathrm{P}$-nodes and their targets.

LEMMA 3.6 (a) $T(\emptyset)=\left\{\pi^{q 0}\right\}$;

(b) $\bigcup_{A \subseteq \Pi \backslash\left\{\pi q^{*}\right\}} T(A)=\Pi$;

(c) $\sigma_{\beta} \subseteq \bigcup_{\substack{1 \subseteq \Pi \backslash\left\{\pi^{\left.q_{*}\right\}}\right\} \\ \alpha \beta \in T(A)}} \operatorname{pow}^{*}\left(\left\{\sigma^{\alpha}: \alpha \in A\right\}\right)$, for all places $\beta \in \Pi$;

(d) $\operatorname{pow}^{*}\left(\left\{\sigma^{\alpha}: \alpha \in A\right\}\right) \subseteq \bigcup_{\beta \in T(A)} \sigma^{\beta}$, for all $P$-nodes $A$.

(e) $|T(A)| \leq n-1$, for all $P$-nodes $A$.

Proof. From Definition 3.4(b), $\pi \in T(\emptyset)$ if and only if $\pi(p)=1$ for all powerset clauses $p=\operatorname{pow}(q)$ in $\phi$. Therefore (a) follows at once from Lemma 3.3(1).

Concerning (b), it is enough to show that every place $\beta \in \Pi$ is target of some P-node A. Let $s \in \sigma^{\beta}$ and consider $A_{s}=\left\{\pi \in \Pi: \sigma^{\pi} \cap s \neq \emptyset\right\}$. As $s \in M p_{v}$, then $s \subseteq M q_{v}$, implying that $A$, is a P-node and that $s \in$ pow $^{*}\left(\left\{\sigma^{\pi}: \pi \in\right.\right.$ 
$\left.\left.A_{s}\right\}\right) \cap \sigma^{\beta}$. By Lemma 3.5, the latter membership implies $\beta \in T\left(A_{s}\right)$, proving (b). Moreover, it follows that

$$
\sigma_{\beta} \subseteq \bigcup_{\cdot \in \sigma^{\beta}} \subseteq \operatorname{pow}^{*}\left(\left\{\sigma^{\pi}: \pi \in A_{\theta}\right\}\right) \subseteq \bigcup_{\substack{A \subseteq M \backslash\left\{\pi \pi^{*}\right\} \\ \dot{\alpha} \in \in T(A)}} \operatorname{pow}^{*}\left(\left\{\sigma^{\alpha}: \alpha \in A\right\}\right),
$$

thus establishing (c).

Concerning (d), let $A$ be a P-node. Since by definition $A \subseteq \Pi \backslash\left\{\pi^{g_{v}}\right\}$, use of Lemma 3.3(3) gives $\alpha\left(q_{v}\right)=1$, for all $\alpha \in A$, i.e. $\bigcup_{\alpha \in A} \sigma^{\alpha} \subseteq M q_{v}$. Hence $\operatorname{pow}^{*}\left(\left\{\sigma^{\alpha}: \alpha \in A\right\}\right) \subseteq \operatorname{pow}\left(\bigcup_{\alpha \in A} \sigma^{\alpha}\right) \subseteq \operatorname{pow}\left(M q_{v}\right)=M q_{v}=\bigcup_{i=1}^{n} \sigma_{i}$. Let $\sigma^{\beta_{1}}, \ldots, \sigma^{\beta_{k}}$ be all parts of the Venn diagram which have nonempty intersection with pow $\left(\left\{\sigma^{\alpha}: \alpha \in A\right\}\right)$. Hence pow $\left(\left\{\sigma^{\alpha}: \alpha \in A\right\}\right) \subseteq \bigcup_{i=1}^{k} \sigma^{\dot{\beta}}$. Moreover, the preceding lemma implies that $\left\{\beta_{1}, \ldots, \beta_{k}\right\} \subseteq T(A)$, proving (d).

Finally, from (a) $|T(0)|=\left|\left\{\pi^{q_{0}}\right\}\right|=1<n-1$, because $n \geq 3$. On the other hand, if $A \neq \emptyset$, then $\alpha\left(q_{0}\right)=0$ for all $\alpha \in A$, so that $\beta\left(p_{0}\right)=0$ for all targets of A. Since $\pi^{q_{0}}\left(p_{0}\right)=1$, it follows $T(A) \subseteq \Pi \backslash\left\{\pi^{q_{0}}\right\}$, thus showing $|T(A)| \leq n-1$ in all cases and concluding the proof of the lemma.

To prepare for the required construction of the map $\pi \mapsto \bar{\pi}$ in (iii), we need a bit of additional terminology.

As above, let $\rho$ be an integer such that

$$
\rho \geq 2 \text { and } 2^{\rho-1}>\rho(n-1)+1
$$

where $n=|\Pi|$.

DEFINITION 3.7 A place $\pi \in I I$ is called $M-\rho$-trapped if $\left|\sigma^{*}\right|<\rho$. A $P$-node $\left\{\alpha_{1}, \ldots, \alpha_{\ell}\right\}$ is called $M$ - $\rho$-trapped if for $i=1, \ldots, \ell$ each $\alpha_{i}$ is trapped.

Remark. In what follows, $M$ - $\rho$-trapped places and $M-\rho$-trapped P-nodes will be referred to simply as trapped places and trapped $P$-nodes, since the model $M$ and the constant $\rho$ will not change in the course of our proof.

DEFINITION 3.8 For places $\alpha, \beta \in \Pi$ we write $\alpha<\beta$ if

$$
\operatorname{rank}\left(\sigma^{\alpha}\right)<\operatorname{rank}\left(\sigma^{\beta}\right) \text {. }
$$

Remark. Since the relation < over II defined above is clearly acyclic, it can be extended to a linear ordering in $\Pi$, which we will designate by the same symbol $<$.

We have the following lemma. 
LEMMA 3.9 (a) $\pi^{q_{0}}=\operatorname{nin} \Pi$ and $\pi^{q_{v}}=\max \Pi$.

(b) Let $\left\{\alpha_{1}, \ldots, \alpha_{\ell}\right\}$ be a nontrapped $P$-node. Then $\left\{\alpha_{1}, \ldots, \alpha_{\ell}\right\}$ has at least one nontrapped target $\beta$ such that $\beta>\max _{i=1, \ldots, l} \alpha_{i}$.

Proof. Concering (a), it is enough to observe that by Lemma $3.3 \operatorname{rank}\left(\sigma^{\pi^{90}}\right)<$ $\operatorname{rank}\left(\sigma^{\pi}\right)$, for all $\pi \in \Pi \backslash\left\{\pi^{q_{0}}\right\}$, and $\operatorname{rank}\left(\sigma^{\pi^{9 *}}\right)>\operatorname{rank}\left(\sigma^{\pi}\right)$, for all $\pi \in \Pi \backslash\left\{\pi^{q_{*}}\right\}$.

To prove (b), let $A=\left\{\alpha_{1}, \ldots, \alpha_{\ell}\right\}$ be a nontrapped P-node. This means that $\left|\sigma^{\alpha_{0}}\right| \geq \rho$ for some $j_{0} \in\{1, \ldots, \ell\}$. Hence it is easy to see that

$$
\left|\left\{t \in \operatorname{pow}^{*}\left(\left\{\sigma^{\alpha_{1}}, \ldots, \sigma^{\alpha_{\ell}}\right\}\right): \operatorname{rank}(t)=\operatorname{rank}\left(\sigma^{\alpha_{1}} \cup \ldots \cup \sigma^{\alpha_{\ell}}\right)\right\}\right| \geq 2^{\rho-1} .
$$

From this inequality, Lemma 3.5 (d) and the pigeon-hole principle we deduce that there must exist a place $\beta \in T(A)$ such that

$$
\left|\left\{t \in \operatorname{pow}^{*}\left(\left\{\sigma^{\alpha_{1}}, \ldots, \sigma^{\alpha_{\ell}}\right\}\right): \operatorname{rank}(t)=\operatorname{rank}\left(\sigma^{\alpha_{1}} \cup \ldots \cup \sigma^{\alpha_{\ell}}\right)\right\} \cap \sigma^{\beta}\right| \geq \rho,
$$

since by Lemma 3.6(d) $2^{\rho-1}>\rho\left(n^{\prime}-1\right)+1>\rho|T(A)|$.

Hence $\left|\sigma^{\beta}\right| \geq \rho$, i.e. $\beta$ is nontrapped, and

$$
\operatorname{rank}\left(\sigma^{\alpha_{i}}\right) \leq \operatorname{rank}\left(\sigma^{\alpha_{1}} \cup \ldots \cup \sigma^{\alpha_{\ell}}\right)<\operatorname{rank}\left(\sigma^{\beta}\right) \text {, for all } i=1, \ldots, \ell,
$$

i.e. $\alpha_{i}<\beta$, for all $i=1, \ldots, \ell$. This completes the proof of the lemma.

Let $=\left\{\alpha_{1}, \ldots, \alpha_{\ell}\right\}$ be a P-node. Then since $U n\left(\left\{\sigma^{\alpha}: \alpha \in A\right\}\right) \in$ pow* $\left(\left\{\sigma^{\alpha}:\right.\right.$ $\alpha \in A\})$, by Lemma 3.6(d) we have $U n\left(\left\{\sigma^{\alpha}: \alpha \in A\right\}\right) \in \sigma^{\top}$, for some place $\tau \in T(A)$. The preceding discussion justifies the following definition.

DEFINITION 3.10 Let A be a P-node. The place $\tau \in T(A)$ such that $U n\left(\left\{\sigma^{\alpha}: \alpha \in A\right\}\right) \in \sigma^{\top}$ is called the principal target of $\mathrm{A}$ and is denoted by $\pi^{A}$.

The following two lemmas state useful properties of principal targets.

LEMMA 3.11 Let $\mathrm{A}$ be a P-node and let $\mathrm{x}$ be a variable in $\phi$ it such that $A=\{\pi \in \Pi: \pi(x)=1\}$. Then the principal target of $\mathrm{A}$ is the place $x^{x}$ at the variable $\mathrm{x}$.

Proof. It is enough to observe that under the hypotheses of the lemma we have $U n\left(\left\{\sigma^{\alpha}: \alpha \in A\right\}\right)=M x \in \sigma^{\pi^{2}}$.

LEMMA 3.12 Let A be a P-node and let $\pi^{A}$ be its principal target. Then $\alpha<$ $\pi^{A}$ for all $\alpha \in A$, where $<$ is the ordering relation introduced in Definition 3.8. 
Proof. By definition we bave $U n\left(\left\{\sigma^{\alpha}: \alpha \in A\right\}\right) \in \sigma^{\pi^{A}}$, so that $\operatorname{rank}\left(\sigma^{\alpha}\right)<$ $\operatorname{rank}\left(\sigma^{\pi^{A}}\right)$, i.e. $\alpha<\pi^{A}$, for all $\alpha \in A$.

We will prove Theorem 3.1 by showing how to use the model $M$ to find an Instantiated Variant of the Association Algorithm (I.V.A.A.). This instantiation, shown below, will consist of

(a) an Initialization Phase, followed by

(b) a Stabilization Loop, which is in turn divided into

(b1) a Blocking Phase, and

(b2) a Propagation Phase;

(c) a procedure called Distribute.

The Instantiated Algorithm which we present uses an arbitrary, perhaps infinite, model of $\phi$ to build a canonical model of $\phi$. This is done in the following way:

(I) For each place $\pi \in \Pi$, the set $\pi$ is initialized to the nullset.

(II) The sets $\bar{\pi}$ are then enlarged progressively (always be calls to the Distribute subprocedure). As enlargement proceeds, an auxiliary 1-1 map $f$ is maintained. The domain of $f$ is always a subset of $\bigcup_{\pi \in \Pi} \bar{\pi}$, and $f$ always maps $\bar{\pi}$ into $\sigma^{\pi}$; moreover $f$ is defined on all of $\bar{\pi}$ as long as $|\bar{\pi}|$ remains less than the critical size $\rho$. We always have $|\bar{\pi}|<\rho$ if $\pi$ is trapped, so that when $\pi$ is trapped $f$ will result to be an 'isomorphism' from $\bar{\pi}$ into $\sigma^{\pi}$. Moreover, the sets $\bar{\pi}$ remain mutually disjoint as they are enlarged.

(III) As the computation proceeds, places $\pi$ successively become 'blocked'; once a place $\pi$ becomes blocked, the set $\bar{\pi}$ ceases to expand. Places become blocked in increasing sequence, according to the or dering relationship $\alpha<$ $\beta$, i.e. if $\alpha<\beta$ then $\alpha$ must already have become blocked when $\beta$ becomes blocked.

(IV) The condition that $\operatorname{pow}^{*}\left(\left\{\bar{\alpha}_{1}, \ldots, \bar{\alpha}_{\ell}\right\}\right) \cap \bar{\beta} \neq \emptyset$ only when $\beta$ is a target of $\left\{\alpha_{1}, \ldots, \alpha_{\ell}\right\}$ (cf. Lemma 3.5 ) is maintained, and moreover the Instantiated Algorithm operates in such a way that elements $t \in$ pow* $\left(\left\{\bar{\alpha}_{1}, \ldots, \bar{\alpha}_{\ell}\right\}\right) \cap \bar{\beta}$ are only introduced by calls Distribute $\left(\alpha_{1}, \ldots, \alpha_{\ell}\right\}$, with $\alpha_{1}, \ldots, \alpha_{\ell}$ as argument list. 
The Main Inductive Lemma (Lemma 3.14) to be proved in the next section will show that the Instantiated Algorithm maintains these invariants, and also will establish various other properties of the Instantiated Algorithm required for the necessary inductive proof. In Section 5 it will be proved that the I.V.A.A. terminates and a bound on its execution lenght will be established. After the rather lengthy detailed proofs of the Main Inductive Lemma and the Termination Lemma, it will become relatively easy to show that the sets $\bar{\pi}$ generated by the Instantiated Algorithm define a model for $\phi$ the rank of each of whose sets satisfies an a priori bound. This additional conclusion, which clearly establishes satisfiability of the decision problem for MLSSP will be proved at the end of this section.

In full detail, the Instantiated Variant of the Association Algorithm is as follows.

$\begin{aligned} \text { [INTTIALIZATION } & \text { Put } \\ \text { PHASE] } & \bar{\pi} \leftarrow \emptyset \\ & \text { for all places } \pi \in \Pi \text {. . } \\ & \text { Put } \\ & f \leftarrow \emptyset . \\ & \text { Mark all places as 'unblocked'. } \\ & \text { Mark all P-nodes as 'unblocked'. } \\ & \text { [END INITIALIZATION PHASE] }\end{aligned}$

[STABILIZATION

$L O O P]$

WHILE there exist unblocked places DO

[BLOCKING

PHASE]

try_block_next_item:

Let $\vartheta_{0}$ be the minimum unblocked place in the ordering < of places.

[Comment: $\vartheta_{0}$ is the next candidate to be blocked.]

IF $\left(\vartheta_{0}\right.$ is trapped and $\left.\left|\overline{\vartheta_{0}}\right|=\left|\sigma^{\vartheta_{0}}\right|\right)$

or $\left(\vartheta_{0}\right.$ is nontrapped and $\left|\overline{\vartheta_{0}}\right| \geq \rho$

and $\left|\overline{\vartheta_{0}} \cap \operatorname{pow}^{*}\left(\left\{\overline{\alpha_{1}}, \ldots, \overline{\alpha_{\ell}}\right\}\right)\right|=\mid \sigma^{\vartheta_{0}} \cap$ $\operatorname{pow}^{*}\left(\left\{\sigma^{\alpha_{1}}, \ldots, \sigma^{\alpha_{2}}\right\}\right) \mid$ for every P-node 
$\left\{\alpha_{1}, \ldots, \alpha_{\ell}\right\}$ which is such that $\left|\overline{\alpha_{j}}\right|<\rho$, for all $j=1, \ldots, \ell$ ) THEN

mark $\vartheta_{0}$ as 'blocked';

FOR ALL P-nodes $\left\{\vartheta_{0}, \vartheta_{1}, \ldots, \vartheta_{k}\right\}$ such that all of $\vartheta_{1}, \ldots, \vartheta_{k}$ are blocked DO mark $\vartheta_{0}$ as 'blocked';

Distribute $\left(\vartheta_{0}, \vartheta_{1}, \ldots, \vartheta_{k}\right)$; (Note: The code for the procedure involved here is shown below.)

END FOR ALL;

GOTO try_block_next_item;

\section{END IF;}

\section{[END BLOCKING PHASE]}

Comment: When the preceding IF test fails, we enter the Propagation Phase.]

[PROPAGATION PHASE]
Pick a node $A=\left\{\alpha_{1}, \ldots, \alpha_{\ell}\right\}$ marked unblocked and such that either $\left(0<\left|\overline{\alpha_{1}}\right|, \ldots,\left|\overline{\alpha_{\ell}}\right| \leq \rho\right.$ and pow $\left.^{*}\left(\left\{\bar{\alpha}_{1}, \ldots, \bar{\alpha}_{\ell}\right\}\right) \backslash \bigcup_{\beta \in T(\boldsymbol{A})} \bar{\beta} \neq \emptyset\right)$ or $\left(\bar{\alpha}_{\boldsymbol{i}} \neq \emptyset\right.$, for $i=1, \ldots, \ell$, and $\left|\widehat{\alpha_{j}}\right| \geq \rho$ for some $\left.j \in\{1, \ldots, \ell\}\right)$, if any such unblocked $\mathrm{P}$-nodes exist, and call Distribute $\left(\alpha_{1}, \ldots, \alpha_{\ell}\right)$; [END PROPAGATION PHASE] END WHILE; [END STABILIZATION LOOP]

[END I.V.A.A.]

Next we show the details of the 'Distribute' subprocedure used in the preceding code.

PROCEDURE Distribute $\left(\alpha_{1}, \ldots, \alpha_{\ell}\right)$;

ASSERT Assertion $A:\left\{\alpha_{1}, \ldots, \alpha_{\ell}\right\}$ is a P-node which is either marked END ASSERT $[$ Assertion $A]$; 
IF $\left|\overline{\alpha_{i}}\right|<\rho$ for all $i=1, \ldots, \ell$ THEN

GOTO update_for_small_alphas;

ELSE [Comment: $\left|\overline{\alpha_{j}}\right| \geq \rho$ for some $j \in\{1, \ldots, \ell\}$ ]

GOTO update_for_one_large_alpha;

END IF;

update_for_small_alphas:

For all $\pi \in \Pi$ let

$$
\Delta_{\pi}=\bar{f}^{-1}\left[\text { pow }^{*}\left(\left\{f\left[\overline{\alpha_{1}}\right], \ldots, f\left[\overline{\alpha_{\ell}}\right]\right\}\right) \cap \sigma^{\pi}\right] \backslash \bigcup_{\beta \equiv T(A)} \bar{\beta}
$$

(where we recall that $\bar{f}$ denotes the function defined on the parts of the domain of $f$ by $\bar{f}(B)=f[B]$, for $B \subseteq \operatorname{dom}(f)$ ).

ASSERT Assertion B:

(B.1) $\Delta_{\pi}=\emptyset$, for all $\pi \in \Pi \backslash T(A)$.

(B.2) $\left\{\Delta_{\pi}: \pi \in \Pi\right\}$ is a partition of $F o w^{*}\left(\left\{\overline{\alpha_{1}}, \ldots \overline{\alpha_{\ell}}\right\}\right) \backslash$ $\bigcup_{\beta \in T(A)} \bar{\beta}$.

(B.3) If $\Delta_{\beta} \neq \emptyset$ then the place $\beta$ is an mblocked target of $\left\{\alpha_{1}, \ldots, \alpha_{\ell}\right\}$, for all $\beta \in \Pi$.

END ASSERT $[$ Assertion $B]$;

Put

$$
\left.f \leftarrow \bar{f} \cup f\right|_{\text {pow }} \cdot\left(\left\{\overline{\alpha_{1}}, \ldots, \overline{\alpha_{\ell}}\right\}\right) \cdot
$$

Also put

$$
\bar{\pi} \leftarrow \bar{\pi} \cup \Delta_{\pi},
$$

for each place $\pi \in \Pi$.

GOTO exit,

update_for_one_large_alpha:

Let $\beta_{1}, \ldots, \beta_{g}, \tau_{1}, \ldots, \tau_{k}, \nu_{1}, \ldots, \nu_{h}$ be all the targets of $\left\{\alpha_{1}, \ldots, \alpha_{\ell}\right\}$, where the $\beta$ 's are the blocked targets, the $\tau$ 's are the unblocked trapped targets and 
the $\nu$ 's are the unblocked nontrapped targets of $\left\{\alpha_{1}, \ldots, \alpha_{\ell}\right\}$, respectively. Let

$$
\begin{gathered}
\Delta^{\prime}=\operatorname{pou}^{*}\left(\left\{\overline{\alpha_{1}}, \ldots, \overline{\alpha_{\ell}}\right\}\right) \backslash \bigcup_{\beta \in T(A)} \bar{\beta} \backslash\left\{\overline{\alpha_{1}} \cup \ldots \cup \overline{\alpha_{\ell}}\right\}, \\
\Delta^{\prime \prime}= \begin{cases}\emptyset & \text { if } A \text { is not blocked } \\
\left\{\overline{\alpha_{1}} \cup \ldots \cup \overline{\alpha_{\ell}}\right\} & \text { if } \mathrm{A} \text { is blocked } \\
\Delta=\Delta^{\prime} \cup \Delta^{\prime \prime} .\end{cases}
\end{gathered}
$$

Also let

$$
\sigma^{A}=\left\{\begin{array}{ll}
\emptyset & \text { if A is blocked } \\
\left\{\sigma^{\alpha_{1}} \cup \ldots \cup \sigma^{\alpha_{\ell}}\right\} & \text { if A is not blocked }
\end{array} .\right.
$$

In addition, for all unblocked trapped targets $\tau_{1}, \ldots, \tau_{k}$ of $A$, let

$$
n_{j}=\left|\sigma^{\tau}, \cap \operatorname{pow}^{*}\left(\left\{\sigma^{\alpha_{1}}, \ldots, \sigma^{\alpha_{\ell}}\right\}\right) \backslash \sigma^{A}\right|-\mid \overline{\tau_{j}} \cap \text { pow }^{*}\left(\left\{\overline{\alpha_{1}}, \ldots, \overline{\alpha_{\ell}}\right\}\right) \mid .
$$

ASSERT Assertion C:

(C.1) $\overline{\alpha_{1}} \cup \ldots \cup \overline{\alpha_{\ell}} \notin \bigcup_{\beta \in T(A)} \bar{\beta}$.

(C.2) $\sigma^{\alpha_{\imath}} \cup \ldots \cup \sigma^{\alpha_{\ell}} \in \sigma^{\star^{\wedge}} \cap$ pow $\left(\left\{\sigma^{\alpha_{1}}, \ldots, \sigma^{\alpha_{\ell}}\right\}\right) \backslash$ range $(f)$, where $\pi^{A}$ is the principal target of the $\mathrm{P}$-node $\boldsymbol{A}$.

(C.3) There exists a partition $\Delta_{\tau_{1}}, \ldots, \Delta_{\tau_{k}}, \Delta_{\nu_{1}}, \ldots, \Delta_{\nu_{h}}$ of the set $\Delta$ such that

(C.3.a) $\left|\Delta_{\tau}\right|=n_{j}$ for each $j=1, \ldots, k$;

(C.3.b) if $\Delta^{\prime} \neq \emptyset$ then $\left|\Delta_{\nu_{i}}\right| \geq \rho$, for each $i=1, \ldots, h$; and

(C.3.c) $\Delta^{\prime \prime} \subseteq \Delta_{\pi} \wedge$.

(C.4) For each $j=1, \ldots, k$,

$$
n_{j}=\left|\sigma^{\tau_{j}} \cap \operatorname{pow}^{*}\left(\left\{\sigma^{\alpha_{1}}, \ldots, \sigma^{\alpha_{\ell}}\right\}\right) \backslash \sigma^{A} \backslash \operatorname{range}(f)\right|
$$

(so that the sets $\Delta_{\tau_{j}}$ and $\sigma^{\tau_{j}} \cap$ pow $\left(\left\{\sigma^{\alpha_{1}}, \ldots, \sigma^{\alpha_{\ell}}\right\}\right) \backslash$ $\sigma^{A} \backslash$ range $(f)$ can be put in 1-1 correspondence).

END ASSERT $[$ Assertion $C]$;

Let $\left\{\Delta_{\pi}: \pi \in \Pi\right\}$ be a partition of the set $\Delta$ such that 
- $\left|\Delta_{r,}\right|=n_{j}$, for each $j=1, \ldots, k$;

- if $\Delta^{\prime} \neq \emptyset$ then $\left|\Delta_{\nu_{1}}\right| \geq \rho$, for each $i=1, \ldots, h$;

- $\Delta^{\prime \prime} \subseteq \Delta_{\pi}{ }^{A}$;

- $\Delta_{\pi}=\emptyset$, if $\pi \in \Pi \backslash\left\{\tau_{1}, \ldots, \tau_{k}, \nu_{1}, \ldots, \nu_{h}\right\}$.

Also, for each $j=1, \ldots, k$, let $f_{\tau}$, denote a 1-1 correspondence between $\Delta_{\tau_{j}}$ and the set $\sigma^{\tau}, \cap$ pow $\left(\left\{\sigma^{\alpha_{1}}, \ldots, \sigma^{\alpha_{\ell}}\right\}\right) \backslash \sigma^{A} \backslash$ range $(f)$, such that if $A$ is blocked and the principal target $\pi^{A}$ of $\mathrm{A}$ is trapped, then $f_{\pi^{A}}\left(\overline{\alpha_{1}} \cup \ldots \cup \overline{\alpha_{l}}\right)$ $=\sigma^{\alpha_{1}} \cup \ldots \cup \sigma^{\alpha_{\ell}}$.

[Comment: From the preceding assertion, it follows immediately that such partition $\left\{\Delta_{\pi}: \pi \in \Pi\right\}$ and functions $f_{\tau_{1}}, \ldots, f_{\tau_{k}}$ exist.

Put

$$
\bar{\pi} \leftarrow \bar{\pi} \cup \Delta_{\pi}
$$

for all $\pi \in \Pi$.

Also put

$$
f \leftarrow f \cup f_{\tau_{1}} \cup \ldots \cup f_{\tau_{k}} .
$$

IF $\left\{\alpha_{1}, \ldots, \alpha_{\ell}\right\}$ is marked unblocked THEN mark $\left\{\alpha_{1}, \ldots, \alpha_{\ell}\right\}$ as 'visited'

END IF;

ASSERT Assertion $D$ : If $\pi$ is trapped, then

$$
\left|\sigma^{\pi} \cap \operatorname{pow}^{*}\left(\left\{\sigma^{\alpha_{1}}, \ldots, \sigma^{\alpha_{\ell}}\right\}\right) \backslash \sigma^{A}\right|=\left|\bar{\pi} \cap p_{0 w}^{*}\left(\left\{\overline{\alpha_{1}}, \ldots, \overline{\alpha_{\ell}}\right\}\right)\right| ;
$$

END ASSERT [Assertion $D]$;

exit:

ASSERT Assertion E:

(E.1) $f$ is a 1-1 function.

(E.2) $\operatorname{dom}(f) \subseteq \bigcup_{\pi \in \Pi} \bar{\pi}$.

(E.3) $f[\bar{\pi}] \subseteq \sigma^{\pi}$, for all $\pi \in \Pi$.

(E.4) If $|\bar{\pi}|<\rho$ then $\bar{\pi} \subseteq \operatorname{dom}(f)$.

(E.5) If $\pi$ is trapped, then $|\bar{\pi}| \leq\left|\sigma^{\pi}\right|$. 
(E.6) $\bar{\alpha} \cap \bar{\beta}=\emptyset$, for all $\alpha, \beta \in \Pi$ with $\alpha \neq \beta$.

(E.7) If $u \in \operatorname{pow}^{*}(\{\bar{\gamma}: \gamma \in \Gamma\}) \cap\left(\cup_{x \in \Pi} \bar{\pi}\right)$, with $\Gamma$ any P-node such that $|\bar{\gamma}|<\rho$ for each $\gamma \in \Gamma$, then $u \in \operatorname{dom}(f), u \subseteq \operatorname{dom}(f)$ and $f(u)=f[u]$.

(E.8) If $u \in \operatorname{pow}^{*}(\{\bar{\gamma}: \gamma \in \Gamma\}) \cap \operatorname{dom}(f)$, with $\Gamma$ any P-node, then $f(u) \in \operatorname{pow}^{\star}\left(\left\{\sigma^{\gamma}: \gamma \in \Gamma\right\}\right)$.

(E.9) If $t \in \operatorname{pow}^{*}\left(\left\{\overline{\gamma_{1}}, \ldots, \overline{\gamma_{h}}\right\}\right) \cap \bar{\delta} \neq \emptyset$, for some places $\gamma_{1}, \ldots, \gamma_{h}, \delta \in \Pi$, then

(E.9.a) $\left\{\gamma_{1}, \ldots, \gamma_{h}\right\}$ is a P-node;

(E.9.b) $\delta$ is a target of $\left\{\gamma_{1}, \ldots, \gamma_{h}\right\}$;

(E.9.c) $t$ must have been introduced into $\bar{\delta}$ during the execution of an earlier call to the procedure Distribute, and the argument to this prior call must have been the same P-node $\left\{\gamma_{1}, \ldots, \gamma_{h}\right\}$.

END ASSERT [Asscrtion E];

END PROCEDURE Distribute.

Remark. As just presented, the I.V.A.A. is still nondeterminstic. Indeed, our Propagation Phase code does not specify how the unblocked P-node is to be chosen among all nodes which satisfy the condition appearing in the code. Moreover, the body of the procedure Distribute does not specify what partition $\left\{\Delta_{\pi}: \pi \in I I\right\}$ of the set $\Delta$ is to be used when a P-node $\left\{\alpha_{1}, \ldots, \alpha_{l}\right\}$, with $\overline{\alpha_{i}} \neq \emptyset$ for all $i=1, \ldots, \ell$ and $\left|\overline{\alpha_{j}}\right| \geq \rho$ for some $j \in\{1, \ldots, \ell\}$, is processed. Nevertheless it is unnecessary to specify these final details since we will show that every possible instantiation of the I.V.A.A. represents an acceptable instance of the Association Algorithm.

The following lemma lists some immediate properties of the I.V.A.A. which can be proved just by inspection of its code.

LEMMA 3.13 For all possible computations of the I.V.A.A. we have:

(a) After the Initialization Phase the sets $\bar{\pi}$ are modified only by calls to the procedure Distribute, and each such modification of a set $\bar{\pi}$ enlarges it.

(b) Once $\left|\bar{\alpha}_{j}\right| \geq \rho$ for some $j \in\{1, \ldots, \ell\}$, a P-node $\left\{\alpha_{1}, \ldots, \alpha_{\ell}\right\}$ can be processed at most once by a call to the procedure Distribute made from the Propagation Phase. 
(c) Evcry P-node can be processed at most once by a call to the procedure Distribute made from the Blocking Phase.

(d) When a $P$-node $\left\{\alpha_{1}, \ldots, \alpha_{\ell}\right\}$ is processed by the procedure Distribute, all places $\beta$ such that $\beta>\alpha_{i}$ for all $i=1, \ldots, \ell$ are unblocked.

(e) Once a place becomes blocked it cannot subsequently become unblocked.

(f) At each call of the procedure Distribute, if all ASSERT-statements are executed successfully then Assertion $E$ is executed.

Let $K$ be an execution of the I.V.A.A., and let $C_{1}, C_{2}, C_{3}, \ldots$ be the sequence of calls to the procedure Distribute arranged in the order in which they occur during the computation $K$.

For each place $\pi \in \Pi$, let $\bar{\pi}^{(r)}$ (resp. $\Delta_{\pi}^{(r)}$ ), $r \geq 1$, designate the value of $\bar{\pi}$ (resp. the value of $\Delta_{\pi}$ ) just after (resp. during) the execution of the $r$-th call $C_{r}$. Analogously, we will denote by $f^{(r)}$ the value of $f$ after completion of $C_{r}$. Moreover, for a given ASSERT-instruction labelled 'Assertion $X$ ' and executed during the processing of the call $C_{r}$, we denote by 'Assertion $\mathrm{X}^{(r)}$ ' the result of substituting in it each program variable by its corresponding value at the time the ASSERT statement is executed. Finally, we put $\bar{\pi}^{(0)}=f^{(0)}=0$, for all $\pi \in \Pi$.

The following basic lemmas, which will be proved in the next sections, express the correctness of the I.V.A.A.

LEMMA 3.14 (Main Inductive Lemma: Partial correctness) All ASSERTstatements encountered during the computation $K$ are executed successfully.

LEMMA 3.15 (Termination) The number of calls $C_{1}, C_{2}, C_{3}, \ldots$ in the computation $K$ is bounded by $(\rho-2)(n-1) 2^{n-2}+3 \cdot 2^{n-1}-2$. Moreover, when the last call to the procedure Distribute is made, all places $\pi \in \Pi$ are already blocked.

Having assumed the validity of Lemma 3.14 and Lemma 3.15, we will prove below that the sets $\bar{\pi}$ produced by the computation $K$ satisfy both conditions (C3) and (C4).

Let $C_{\xi}$ be the last call to Distribute in the computation K. For simplicity, we will often write $\bar{\pi}$ in place of $\bar{\pi}^{(\xi)}$, for all $\pi \in \Pi$, and $f$ in place of $f^{(\xi)}$.

In order to prove that condition (C3.a) is fulfilled, it is enough to observe that since all places $\pi \in \Pi$ are blocked at the end of the computation $K$, then either $|\bar{\pi}|=\left|\sigma^{\pi}\right|$, if $\pi$ is trapped, or $|\bar{\pi}| \geq \rho$, if $\pi$ is nontrapped, and in any case $\bar{\pi} \neq \emptyset$. 
Condition (C3.b) follows immediately from Assertion (E.8) $)^{(\xi)}$.

To prove that condition (C3.C) is satisfied, we will need the lemma below.

LEMMA 3.16 (1) Let $\left|\bar{\alpha}_{i}^{(r-1)}\right|<\rho$, for $i=1, \ldots, \ell$ and $1 \leq r \leq \xi$. Then

$$
\begin{aligned}
& \overline{f^{(r-1)}}\left[\text { pow w}^{*}\left(\left\{{\overline{\alpha_{1}}}^{(r-1)}, \ldots,{\overline{\alpha_{l}}}^{(r-1)}\right\}\right)\right] \\
= & \operatorname{pow}^{*}\left(\left\{f^{(r-1)}\left[{\overline{\alpha_{1}}}^{(r-1)}\right], \ldots, f^{(r-1)}\left[{\bar{\alpha} \ell^{(r-1)}}^{(r)}\right\}\right\}\right)
\end{aligned}
$$

(2) Let $C_{\tau}$ be the call Distribute $\left(\alpha_{1}, \ldots, \alpha_{\ell}\right)$. Then

$$
\bigcup_{x \in \Pi}\left(\bar{\pi}^{(r)} \backslash \bar{\pi}^{(r-1)}\right) \subseteq \operatorname{pow}^{*}\left(\left\{{\overline{\alpha_{1}}}^{(r-1)}, \ldots, \bar{\alpha}_{\ell}^{(r-1)}\right\}\right) .
$$

(3) $\operatorname{pow}^{\bullet}\left(\left\{\overline{\alpha_{1}}, \ldots, \overline{\alpha_{\ell}}\right\}\right) \subseteq \bigcup_{\beta \in T(A)} \bar{\beta}$, for all P-nodes $A=\left\{\alpha_{1}, \ldots, \alpha_{\ell}\right\}$.

Proof. Concerning (1), if $\left|{\widehat{\alpha_{i}}}^{(r-1)}\right|<\rho$, for $i=1, \ldots, \ell$, then from Assertion $E^{(r-1)}, \bar{\alpha}_{i}^{(r-1)} \subseteq \operatorname{dom}\left(f^{(r-1)}\right), i=1, \ldots, \ell$, and $f^{(r-1)}$ is 1-1. Therefore Lemma 2.7(b.2) implies (1).

Assertions (B.2) $)^{(r)}$ and (C.3) $)^{(r)}$ imply (2).

Finally to prove (3) let $C_{r_{0}}$ be the last call to Distribute with argument the P-node $A=\left\{\alpha_{1}, \ldots, \alpha_{\ell}\right\}$. Then (3) is an immediate consequence of Assertion $(\mathrm{B} .2)^{\left(\mathrm{r}_{0}\right)}$ and $(\mathrm{C} .3)^{\left(\mathrm{r}_{0}\right)}$.

We are now ready to prove (C3.c). To this end, let $x$ be a variable in $V \backslash\left\{p_{v}\right\}$, and let $\left\{\alpha_{1}, \ldots, \alpha_{\ell}\right\}=\{x \in \Pi: \pi(x)=1\}$. Notice that by Lemma 3.2 the set $A=\left\{\alpha_{1}, \ldots, \alpha_{\ell}\right\}$ is a $\mathrm{P}$-node. Therefore clause (3) of the preceding lemma implies that $\bigcup_{\pi(x)=1} \bar{\pi}=\bigcup_{i=1}^{l} \overline{\alpha_{i}} \in \bar{\beta}$, for some place $\beta \in T(A)$. Let $C_{r_{0}}$ be the call Distribute $\left(\alpha_{1}, \ldots, \alpha_{\ell}\right)$ during whose execution the element $\bigcup_{\pi(x)=1} \bar{\pi}$ is introduced into $\bar{\beta}$. If $\left|\overline{\alpha_{i}}\right|<\rho$ for all $i=1, \ldots, \ell$, then all places $\alpha_{i}$ are trapped. Therefore by combining Assertions (E.4 $)^{(\xi)},(\text { E.3 })^{(\xi)}$, and the fact that all the $\alpha_{i}$ 's are blocked, we obtain

$$
\begin{aligned}
& \overline{f^{\left(r_{0}-1\right)}}\left({\overline{\alpha_{1}}}^{\left(r_{0}-1\right)} \cup \ldots \cup{\overline{\alpha_{\ell}}}^{\left(r_{0}-1\right)}\right) \\
& =f\left[\overline{\alpha_{1}} \cup \ldots \cup \overline{\alpha_{\ell}}\right] \\
& =f\left[\overline{\alpha_{1}}\right] \cup \ldots \cup f\left[\overline{\alpha_{\ell}}\right] \\
& =\sigma^{\alpha_{1}} \cup \ldots \cup \sigma^{\alpha_{t}} \\
& \in \operatorname{pow}^{*}\left(\left\{f\left[\overline{\alpha_{1}}\right], \ldots, f\left[\overline{\alpha_{l}}\right]\right\}\right) \cap \sigma^{\pi^{*}} \\
& =\operatorname{pow}^{*}\left(\left\{f^{\left(r_{0}-1\right)}\left[{\overline{\alpha_{1}}}^{\left(r_{0}-1\right)}\right], \ldots, f^{\left(r_{0}-1\right)}\left[{\overline{\alpha_{\ell}}}^{\left(r_{0}-1\right)}\right]\right\}\right) \cap \sigma^{\pi^{*}},
\end{aligned}
$$


which shows that ${\overline{\alpha_{1}}}^{\left(r_{0}-1\right)} \cup \ldots \cup \overline{\alpha_{\ell}}\left(r_{0}-1\right) \in \Delta_{x^{x}}^{\left(r_{0}\right)} \subseteq \overline{\pi^{x}}$, i.e. $\beta \equiv \pi^{x}$. On the other hand, if $\left|\overline{\alpha_{j}}\right| \geq \rho$ for some $j \in\{1, \ldots, \ell\}$, our conclusion follows from Assertion (C3.c) ${ }^{\left(r_{0}\right)}$, thus fully establishing condition (C3.c).

Next we prove that condition (C3.d) is also satisfied, i.e. that if $p=\operatorname{pow}(q)$ is a powerset clause in $\phi$, then $\bigcup_{\alpha(p)=1} \bar{\alpha}=$ pow $\left(\bigcup_{\beta(q)=1} \bar{\beta}\right)$. Let $\alpha \in \Pi$ such that $\alpha(p)=1$. Lemma 3.13(a) implies that for each $t \in \bar{\alpha}$ there exists a P-node $B_{t}$ such that $\alpha \in T\left(B_{t}\right)$ and $t \in \operatorname{pow}^{*}\left(\left\{\bar{\beta}: \beta \in B_{t}\right\}\right)$. Notice also that $\beta(q)=1$ for each $\beta \in B_{t}$, so that $\bigcup_{\alpha(p)=1} \bar{\alpha} \subseteq$ pow $\left(\bigcup_{\beta(q)=1} \bar{\beta}\right)$. To show the converse inclusion, let $t \in \operatorname{pow}\left(\bigcup_{\beta(q)=1} \bar{\beta}\right)$. Then $t \in$ pow $^{*}\left(\left\{\overline{\beta_{1}}, \ldots, \overline{\beta_{k}}\right\}\right)$ for some places $\beta_{1}, \ldots, \beta_{k}$, with $\beta_{j}(q)=1$ for all $j=1, \ldots, k$. In view of Lemma 3.16(3) we have pow* $\left(\left\{\overline{\beta_{1}}, \ldots, \overline{\beta_{k}}\right\}\right) \subseteq \bigcup_{\alpha \in T(B)} \bar{\alpha} \subseteq \bigcup_{\alpha(p)=1} \bar{\alpha}$, where $B=\left\{\beta_{1}, \ldots, \beta_{k}\right\}$. Therefore pow $\left(\bigcup_{\beta(q)=1} \bar{\beta}\right) \subseteq \bigcup_{\alpha(p)=1} \bar{\alpha}$, thus proving (C3.d).

Finally, to complete the proof of condition (C3) we only need to verify (C3.e). To this end, let $x=\{y\}$ be a singleton clause in $\phi$. From (C3.c) and Lemma 3.3(5), we have $\bigcup_{\beta(y)=1} \bar{\beta} \in \overline{\pi^{y}}=\bigcup_{\alpha(x)=1} \bar{\alpha}$. But $\left|\sigma^{\pi^{y}}\right|=1$, i.e. $\pi^{y}$ is trapped. Therefore Assertion (E.5) $)^{(\xi)}$ implies $\left|\overline{\pi^{y}}\right|=1$, which in turn yields $\bigcup_{\alpha(x)=1} \bar{\alpha}=\left\{\bigcup_{\beta(y)=1} \bar{\beta}\right\}$. This completes the proof of the necessity of condition (C3).

In view of Lemma 3.15 , to prove (C4) it is enough to show that each call to the procedure Distribute is an instantiation of Step 2 of the Association Algorithm. But this follows plainly from Lemma 3.16(2). Hence, up to the proofs of Lemmas 3.14 and 3.15 (which will be provided in the following sections), the proof of the necessity of conditions (C1)-(C4) of Theorem 3.1 is completed.

Notice that for each conjunction $\phi$ in the class MLSSP" there are only finitely many and $a$ priori determinable sets of places of $\phi$ and mappings $x \mapsto \pi^{x}$ and $\pi \mapsto \bar{\pi}$ as in (i)-(iii) of Theorem 3.1 and satisfying conditions (C1)-(C4). In other words, Theorem 3.1 contains a decidability test for the MLSSP" injective satisfiability problem. Therefore, Lemmas $2.2,2.3$ and the discussion at the beginning of the preceding section yield the following corollary.

COROLLARY 3.17 The class of formulae MLSSP has a solvable satisfiability problem.

Actually, Theorem 3.1 implies a slightly stronger result.

COROLLARY 3.18 Let $\phi$ be a conjunction in MLSSP" in which only m distinct variables occur. Then $\phi$ is satisfiable if and only if it has a model of rank at most

$$
\left(m+2\left\lceil\ell g_{2} m\right\rceil-2\right) 2^{2^{m-1}+m-2}+3 \cdot 2^{2^{m-1}}-2 .
$$


Proof. The above condition for the satisfiability of $\phi$ is trivially sufficient.

Conversely, if $\phi$ is satisfiable, by Theorem 3.1 there exist $\Pi, \boldsymbol{x} \mapsto \pi^{\boldsymbol{x}}$ and $\pi \mapsto \bar{\pi}$ as from (i)-(iii) and satisfying (C1)-(C4). As shown in the sufficiency proof of Theorem 3.1 , the assignment $M^{*} x=\bigcup_{\pi(x)=1} \bar{\pi}$, for all $x$ occurring in $\phi$, is a model of $\phi$.

We will prove the corollary by showing that for every $x$ in $\phi$,

$$
\operatorname{rank}\left(M^{*} x\right) \leq\left(m+2\left\lceil\ell g_{2} m\right\rceil-2\right) 2^{2^{m-1}+m-2}+3 \cdot 2^{2^{m-1}}-2 .
$$

For this, observe that by Lemma 3.3(4), $n=|\Pi| \leq 2^{m-1}+1$. Moreover, if we put $\rho=m+2\left[\ell g_{2} m\right\rceil$, since $m \geq 4$ then $\rho \geq 2$, so that $\rho \geq 2$ and $2^{\rho-1}>\rho(n-1)+1$ are both satisfied. Therefore we may assume that the $\rho$ appearing in (C4) has been chosen in this way.

During each execution of Step 2 of the Association Algorithm, the rank of any set $\bar{\pi}$ of maximal rank is augmented by at most 1 . Since by (C4) $\pi \mapsto \bar{\pi}$ is produced by a computation of the Association Algorithm in which Step 2 is executed at most $(\rho-2)(n-1) 2^{n-2}+3 \cdot 2^{n-1}-2$ times, the corollary follows.

An immediate consequence is the following.

COROLLARY 3.19 Given a conjunction $\phi$ in MLSSP", $\phi$ is satisfiable if and only if it is hereditarily finitely satisfiable.

In the next section we will prove the Main Inductive Lemma.

\section{Proof of the Main Inductive Lemma}

In this section we will prove the partial correctness of the I.V.A.A. .

As in the previous section, let $K$ be an execution of the I.V.A.A., and let $C_{1}, C_{2}, C_{3}, \ldots$ be the sequence of calls to the procedure Distribute, arranged in the order in which they occur during the computation $K$. We will also make use of the notation $\bar{\pi}^{(r)}, \Delta_{\pi}^{(r)}, f^{(r)}$, Assertion $\mathrm{X}^{(r)}$ as explained in the previous section. For the sake of completeness we restate the Main Inductive Lemma.

LEMMA 3.14 (Main Inductive Lemma: Partial Correctness) All ASSERTstatements encountered during the computation $\mathrm{K}$ are executed successfully.

Proof. We will proceed by induction on $r \geq 1$, by proving that all ASSERTstatements executed during the processing of the call $C_{r}$ are completed successfully, i.e. they hold at the time they are encountered. 
Base case $r=1$. Notice that $f^{(0)}=\emptyset$ and $\bar{\pi}^{(0)}=\emptyset$ for all places $\pi \epsilon$ ПI. Moreover, when the call $C_{1}$ is made, all places and P-nodes are marked unblocked. Indeed all places and $\mathrm{P}$-nodes are marked unblocked during the Initialization Phase, so that it is enough to show that no place and no P-node can become blocked during the subsequent Blocking Phase. Observe that by Lemma 3.9(a) $\pi^{90}$ is the minimum unblocked place after the execution of the Initialization Phase. But $\overline{\pi^{90}}(0)=\emptyset$, so that the $I F$-test of the Blocking Phase fails. As $\bar{\pi}^{(0)}=\emptyset$, for all $\pi \in \Pi$, the only P-node satisfying the conditions in the Propagation Phase is the empty P-node. Indeed, it is immediate to see (cf. Lemma 2.6(a)) that pow ${ }^{*}(\emptyset) \backslash \bigcup_{\beta \in T(\theta)} \bar{\beta}^{(0)}=\{\emptyset\}$. Therefore $C_{1}$ is the call Distribute( $(\emptyset)$. Assertion $A^{(0)}$ is plain. Notice that $\left|\bar{\alpha}^{(0)}\right|<\rho$, for all $\alpha$ in the empty $\mathrm{P}$-node, is vacuously true, so that the empty $\mathrm{P}$-node is processed at updatefor_small_alphas. It is immediate to see that

$$
\Delta_{\pi}^{(1)}= \begin{cases}\emptyset & \text { if } \pi \neq \pi^{90} \\ \{\emptyset\} & \text { if } \pi=\pi^{90}\end{cases}
$$

and obviously Assertion $\mathrm{B}^{(1)}$ is true (cf. Lemma 3.6(a)). After the execution of the assignment statements, we have

$$
\bar{\pi}^{(1)}=\left\{\begin{array}{ll}
\emptyset & \text { if } \pi \neq \pi^{90} \\
\{\emptyset\} & \text { if } \pi=\pi^{90}
\end{array} \text { and } f^{(1)}=\{(\emptyset, \emptyset)\}\right.
$$

To complete the proof in the base case, we only have to prove that Assertion $\mathrm{E}^{(1)}$ holds. Assertions $(\mathrm{E} .1)^{(1)},(\text { E.2 })^{(1)},(\text { E.4 })^{(1)}-(\mathrm{E} .9)^{(1)}$ are immediate. Concerning Assertion (E.3) $)^{(1)}$ it is enough to observe that by Lemma 3.3(1), $\sigma^{\pi^{90}}=\{\emptyset\}=\operatorname{range}\left(f^{(1)}\right)$. This completes our first induction step.

Inductive step. Next we assume that all ASSERT-statements encountered before the execution of the call $C_{r_{0}}$, with $r_{0}>1$, are completed successfully, and we prove that all ASSERT-statements met during the processing of the call $C_{r_{0}}$ are valid as well.

Let $C_{r_{0}}$ be the call Distribute $\left(\alpha_{1}, \ldots, \alpha_{\ell}\right)$. Clearly the P-node $A=\left\{\alpha_{1}, \ldots \alpha_{\ell}\right\}$ cannot be the empty P-node. Notice also that according to whether the call $C_{r_{0}}$ is made from the Blocking Phase or from the Propagation Phase, we have respectively that either $A$ is blocked or $A$ is unblocked. In any case $A$ cannot be marked visited, showing that Assertion $\mathrm{A}^{\left(r_{0}\right)}$ holds.

To prove the validity of the remaining assertions, we will distinguish two cases according to whether $\left|{\widehat{\alpha_{i}}}^{\left(r_{0}-1\right)}\right|<\rho$ for all $i=1, \ldots, \ell$, or $\left|{\overline{\alpha_{i}}}^{\left(r_{0}-1\right)}\right| \geq \rho$ for some $j_{0} \in\{1, \ldots, \ell\}$. 
Case: $\left|\bar{\alpha}_{i}^{-\left(r_{0}-1\right)}\right|<\rho$ for all $i=1, \ldots, \ell$. In this case we have

$$
\begin{gathered}
\Delta_{\pi}^{\left(r_{0}\right)=} \overline{\left(\overline{f^{\left(r_{0}-1\right)}}\right)^{-1}[\text { pow }}\left(\left\{f^{\left(r_{0}-1\right)}\left[{\overline{\alpha_{1}}}^{\left(\tau_{0}-1\right)}\right], \ldots\right.\right. \\
\left.\left.\left.\ldots, f^{\left(r_{0}-1\right)}\left[{\overline{\alpha_{\ell}}}^{\left(\tau_{0}-1\right)}\right]\right\}\right) \cap \sigma^{\pi}\right] \backslash \bigcup_{\beta \in T(A)} \bar{\beta}^{\left(r_{0}-1\right)} .
\end{gathered}
$$

for all $\pi \in \Pi$.

The following lemma states some useful properties.

LEMMA 4.1 Let $\gamma_{1}, \ldots, \gamma_{k}$ be places of $\phi$ such that $\left|\bar{\gamma}_{j}^{\left(r_{0}-1\right)}\right|<\rho$ for $j=$ $1, \ldots, k$. Then

(i) $\overline{\gamma_{j}}\left(r_{0}-1\right) \subseteq \operatorname{dom}\left(f^{\left(r_{0}-1\right)}\right)$, for each $j=1, \ldots, k$;

(ii) $f^{\left(r_{0}-1\right)}\left[\bar{\gamma}_{j}^{\left(r_{0}-1\right)}\right] \subseteq \sigma^{\gamma}$, for each $j=1, \ldots, k$;

(iii) $\operatorname{pow}^{*}\left(\left\{f^{\left(r_{0}-1\right)}\left[{\overline{\gamma_{1}}}^{\left(r_{0}-1\right)}\right], \ldots, f^{\left(r_{0}-1\right)}\left[{\overline{\gamma_{k}}}^{\left(r_{0}-1\right)}\right]\right\}\right) \subseteq \operatorname{pow}^{*}\left(\left\{\sigma^{\gamma_{1}}, \ldots, \sigma^{\gamma_{k}}\right\}\right)$.

Proof. By inductive hypothesis Assertion $\mathrm{E}^{\left(\mathrm{r}_{0}-1\right)}$ we have plainly (i) and (ii). By Lemma 2.6(c) and (ii) we have also (iii).

Let $\pi \in \Pi \backslash T(A)$. Then, by Lemma 3.6(d), pow* $\left(\left\{\sigma^{\alpha}: \alpha \in A\right\}\right) \cap \sigma^{\pi}=\emptyset$, which by (iii) of the preceding lemma yields $\Delta_{\pi}^{\left(r_{0}\right)}=\emptyset$, thus proving Assertion $(\mathrm{B} .1)^{\left(r_{0}\right)}$.

Next we prove that $(B .2)^{\left(r_{0}\right)}$ is also satisfied. First of all we notice that as $f^{\left(r_{0}-1\right)}$ is $1-1$, the sets $\Delta_{\pi}^{\left(r_{0}\right)}$ are pairwise disjoint, since so are the sets $\sigma^{\pi}$. Moreover from Lemma 2.7 , Lemma 4.1 , and the injectivity of $f^{\left(r_{0}-1\right)}$, we have

$$
\begin{aligned}
& \bigcup_{\pi \in \Pi} \Delta_{\pi}^{\left(r_{0}\right)}=\bigcup_{\pi \in \Pi}\left(\overline{f^{\left(r_{0}-1\right)}}\right)^{-1}\left[p o w ^ { * } \left(\left\{f^{\left(r_{0}-1\right)}\left[{\overline{\alpha_{1}}}^{\left(r_{0}-1\right)}\right], \ldots\right.\right.\right. \\
& \left.\left.\left.\ldots, f^{\left(r_{0}-1\right)}\left[\overline{\alpha \ell}^{\left(r_{0}-1\right)}\right]\right\}\right) \cap \sigma^{\pi}\right] \backslash \bigcup_{\beta \in T(A)} \bar{\beta}^{\left(r_{0}-1\right)} \\
& =\left(\overline{f^{\left(r_{0}-1\right)}}\right)^{-1}\left[p o w^{*}\left(\left\{f^{\left(r_{0}-1\right)}\left[{\overline{\alpha_{1}}}^{\left(r_{0}-1\right)}\right], \ldots, f^{\left(r_{0}-1\right)}\left[{\overline{\alpha_{\ell}}}^{\left(r_{0}-1\right)}\right]\right\}\right)\right. \\
& \left.\cap \bigcup_{\pi \in \Pi} \sigma^{\pi}\right] \backslash \cdot \bigcup_{\beta \in T(A)} \bar{\beta}^{\left(r_{0}-1\right)} \\
& =\left(\overline{f^{\left(r_{0}-1\right)}}\right)^{-1}\left[p o w^{*}\left(\left\{f^{\left(r_{0}-1\right)}\left[{\overline{\alpha_{1}}}^{\left(r_{0}-1\right)}\right], \ldots, f^{\left(r_{0}-1\right)}\left[{\overline{\alpha_{\ell}}}^{\left(r_{0}-1\right)}\right]\right\}\right)\right. \\
& \backslash \bigcup_{\beta \in T(A)} \bar{\beta}^{\left(r_{0}-1\right)} \\
& =p o w^{*}\left(\left\{{\overline{\alpha_{1}}}^{\left(r_{0}-1\right)}, \ldots,{\overline{\alpha_{\ell}}}^{\left(r_{0}-1\right)}\right\}\right) \backslash \bigcup_{\beta \in T(A)} \bar{\beta}^{\left(r_{0}-1\right)} .
\end{aligned}
$$


This completes the proof of Assertion (B.2) ${ }^{\left(r_{0}\right)}$.

Finally, to prove $(B .3)^{\left(r_{0}\right)}$, let $\beta_{0}$ be a place of $\phi$ such that $\Delta_{\beta_{0}}^{\left(r_{0}\right)} \neq \emptyset$. From $(\mathrm{B} .1)^{\left(r_{0}\right)}$ we have $\beta_{0} \in T(A)$, so that we only need to show that the place $\beta_{0}$ is unblocked at the time the call $C_{r_{0}}$ has been made. We distinguish two subcases according to whether $\beta_{0}$ is trapped or not.

Subcase: $\beta_{0}$ is trapped. If $\beta_{0}$ were blocked when the call $C_{\tau_{0}}$ is made, then $\left|{\overline{\beta_{0}}}^{\left(r_{0}-1\right)}\right|=\left|\sigma^{\beta_{0}}\right|<\rho$, which by Lemma 4.1 gives $f^{\left(r_{0}-1\right)}\left[{\overline{\beta_{0}}}^{\left(r_{0}-1\right)}\right]=\sigma^{\beta_{0}}$. Therefore, again from Lemma 4.1 and the inductive hypothesis $(E .8)^{\left(r_{0}-1\right)}$, we would have

$$
\begin{aligned}
\Delta_{\beta_{0}}^{\left(r_{0}\right)} & \subseteq\left(\overline{f^{\left(r_{0}-1\right)}}\right)^{-1}\left[\overline{f^{\left(r_{0}-1\right)}}\left[\text { pow }\left(\left\{{\overline{\alpha_{1}}}^{\left(r_{0}-1\right)}, \ldots,{\overline{\alpha_{\ell}}}^{\left(r_{0}-1\right)}\right\}\right)\right]\right. \\
& \left.\cap f^{\left(r_{0}-1\right)}\left[{\overline{\beta_{0}}}^{\left(r_{0}-1\right)}\right]\right] \\
& \subseteq\left(f^{\left(r_{0}-1\right)}\right)^{-1} f^{\left(r_{0}-1\right)}\left[{\overline{\beta_{0}}}^{\left(r_{0}-1\right)}\right] \\
& ={\overline{\beta_{0}}}^{\left(r_{0}-1\right)} \\
& \subseteq \bigcup_{\beta \in T(A)} \bar{\beta}^{\left(r_{0}-1\right)} .
\end{aligned}
$$

The above inclusion chain implies that $\Delta_{\beta_{0}}^{\left(r_{0}\right)}=\emptyset$, contradicting our assumption $\Delta_{\beta_{0}}^{\left(r_{0}\right)} \neq \emptyset$, and consequently showing that the place $\beta_{0}$ must be unblocked, at least in the case in which $\beta_{0}$ is trapped.

Subcase: $\beta_{0}$ is nontrapped. Suppose that $\beta_{0}$ has become blocked just before the call $C_{\tau^{\prime}}$, with $1 \leq r^{\prime} \leq r_{0}$. Therefore

$$
\left|p o w^{*}\left(\left\{{\overline{\alpha_{1}}}^{\left(r^{\prime}-1\right)}, \ldots,{\overline{\alpha_{l}}}^{\left(r^{\prime}-1\right)}\right\}\right) \cap{\overline{\beta_{0}}}^{\left(r^{\prime}-1\right)}\right|=\left|p o w^{*}\left(\left\{\sigma^{\alpha_{1}}, \ldots, \sigma^{\alpha_{\ell}}\right\}\right) \cap \sigma^{\beta_{0}}\right|
$$

since $\overline{\alpha_{i}}\left(r^{\prime}-1\right) \subseteq{\overline{\alpha_{i}}}^{\left(r_{0}-1\right)}$ for all $i=1, \ldots, \ell$.

Notice also that

$$
\begin{gathered}
\operatorname{pow}^{*}\left(\left\{{\overline{\alpha_{1}}}^{\left(r^{\prime}-1\right)}, \ldots,{\overline{\alpha_{\ell}}}^{\left(r^{\prime}-1\right)}\right\}\right) \cap{\overline{\beta_{0}}}^{\left(r^{\prime}-1\right)} \\
\subseteq \operatorname{pow}^{*}\left(\left\{{\overline{\alpha_{1}}}^{\left(r_{0}-1\right)}, \ldots,{\overline{\alpha_{\ell}}}^{\left(r_{0}-1\right)}\right\}\right) \cap{\overline{\beta_{0}}}^{\left(r_{0}-1\right)} .
\end{gathered}
$$

Moreover, let $u \in$ pow $\left(\left\{{\overline{\alpha_{1}}}^{\left(r_{0}-1\right)}, \ldots,{\overline{\alpha_{l}}}^{\left(r_{0}-1\right)}\right\}\right) \cap{\overline{\beta_{0}}}^{\left(r_{0}-1\right)}$, and let $C_{r \prime \prime}$ with $1 \leq r^{\prime \prime} \leq r_{0}$, be the call Distribute $\left(\alpha_{1}, \ldots, \alpha_{\ell}\right)$ during whose execution the element $u$ is introduced into $\overline{\beta_{0}}$ (cf. inductive hypothesis $(E .9)^{\left(r_{0}-1\right)}$ ). Since

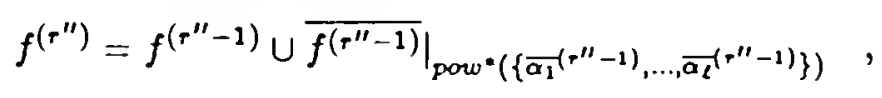


we have

$$
f^{\left(r_{0}-1\right)}(u)=f^{\left(r^{\prime \prime}\right)}(u)=\overline{f^{\left(r^{\prime \prime}-1\right)}}(u)=\overline{f^{\left(r_{0}-1\right)}}(u) .
$$

Therefore $\overline{f^{\left(r_{0}-1\right)}}(u)=f^{\left(r_{0}-1\right)}(u) \in f^{\left(r_{0}-1\right)}\left[{\overline{\beta_{0}}}^{\left(r_{0}-1\right)}\right] \subseteq \sigma^{\beta_{0}}$, so that

$$
\overline{f^{\left(r_{0}-1\right)}}(u) \in \overline{f^{\left(r_{0}-1\right)}}\left[\text { pow* }\left(\left\{{\overline{\alpha_{1}}}^{\left(r_{0}-1\right)}, \ldots,{\overline{\alpha_{\ell}}}^{\left(r_{0}-1\right)}\right\}\right)\right] \cap \sigma^{\beta_{0}},
$$

thus proving

$$
\begin{aligned}
& \overline{f^{\left(r_{0}-1\right)}}\left(\text { pow }^{*}\left(\left\{{\overline{\alpha_{1}}}^{\left(r_{0}-1\right)}, \ldots, \overline{\alpha_{l}}{ }^{\left(r_{0}-1\right)}\right\}\right) \cap{\overline{\beta_{0}}}^{\left(r_{0}-1\right)}\right\} \\
& \subseteq \overline{f^{\left(r_{0}-1\right)}}\left[p \circ w^{*}\left(\left\{{\overline{\alpha_{1}}}^{\left(r_{0}-1\right)}, \ldots,{\overline{\alpha_{\ell}}}^{\left(r_{0}-1\right)}\right\}\right)\right] \cap \sigma^{\beta_{0}} \text {. }
\end{aligned}
$$

From Lemmas 2.7 and 5.1 we have

$$
\begin{aligned}
& \overline{f^{\left(r_{0}-1\right)}}\left[\operatorname{pow}^{*}\left(\left\{{\overline{\alpha_{1}}}^{\left(r_{0}-1\right)}, \ldots, \bar{\alpha}_{\ell}^{\left(r_{0}-1\right)}\right\}\right)\right] \\
\subseteq & \operatorname{pow}^{*}\left(\left\{f^{\left(r_{0}-1\right)}\left[{\overline{\alpha_{1}}}^{\left(r_{0}-1\right)}\right\}, \ldots, f^{\left(r_{0}-1\right)}\left[{\overline{\alpha_{\ell}}}^{\left(r_{0}-1\right)}\right]\right\}\right) \\
\subseteq & \operatorname{pow}^{*}\left(\left\{\sigma^{\alpha_{1}}, \ldots, \sigma^{\alpha_{l}}\right\}\right) .
\end{aligned}
$$

Hence

$$
\begin{aligned}
& \overline{f^{\left(r_{0}-1\right)}}\left[\text { pow* }\left(\left\{\overline{\alpha_{1}}\left(r^{\prime}-1\right), \ldots, \overline{\alpha_{\ell}}\left(r^{\prime}-1\right)\right\}\right) \cap{\overline{\beta_{0}}}^{\left(r^{\prime}-1\right)}\right\} \\
& \subseteq \overline{f^{\left(r_{0}-1\right)}}\left[\text { pow }^{*}\left(\left\{{\overline{\alpha_{1}}}^{\left(r_{0}-1\right)}, \ldots, \overline{\alpha_{l}}\left(r_{0}-1\right)\right\}\right) \cap{\overline{\beta_{0}}}^{\left(r_{0}-1\right)}\right] \\
& \subseteq \operatorname{pow}^{*}\left(\left\{f^{\left(r_{0}-1\right)}\left[{\overline{\alpha_{1}}}^{\left(r_{0}-1\right)}\right], \ldots, f^{\left(\tau_{0}-1\right)}\left[{\overline{\alpha_{\ell}}}^{\left(r_{0}-1\right)}\right]\right\}\right) \cap \sigma^{\beta_{0}} \\
& \subseteq \operatorname{pow}^{*}\left(\left\{\sigma^{\alpha_{1}}, \ldots, \sigma^{\alpha_{\ell}}\right\}\right) \cap \sigma^{\beta_{0}} \text {. }
\end{aligned}
$$

As $\overline{f^{\left(\tau_{0}-1\right)}}$ is injective and pow $\left(\left\{{\overline{\alpha_{1}}}^{\left(r^{\prime}-1\right)}, \ldots, \overline{\alpha \ell}^{\left(r^{\prime}-1\right)}\right\}\right)$ has finite cardinality, the latter chain of inclusions combined with above cardinality equality yields

$$
\begin{aligned}
& \overline{f^{\left(r_{0}-1\right)}}\left[\text { pow }\left(\left\{{\overline{\alpha_{1}}}^{\left(r_{0}-1\right)}, \ldots,{\overline{\alpha_{\ell}}}^{\left(r_{0}-1\right)}\right\}\right) \cap{\overline{\beta_{0}}}^{\left(r_{0}-1\right)}\right\} \\
= & \operatorname{pow}^{*}\left(\left\{f^{\left(r_{0}-1\right)}\left[{\overline{\alpha_{1}}}^{\left(r_{0}-1\right)}\right], \ldots, f^{\left(r_{0}-1\right)}\left[\bar{\alpha}^{\left(r_{0}-1\right)}\right]\right\}\right) \cap \sigma^{\beta_{0}} .
\end{aligned}
$$

and therefore

$$
\begin{aligned}
\Delta_{\beta_{0}}^{\left(r_{0}\right)} & \subseteq{\overline{f^{\left(r_{0}-1\right)}}}^{-1}\left[\operatorname{pow}^{*}\left(\left\{f^{\left(r_{0}-1\right)}\left[{\overline{\alpha_{1}}}^{\left(r_{0}-1\right)}\right], \ldots, f^{\left(r_{0}-1\right)}\left[{\overline{\alpha_{\ell}}}^{\left(r_{0}-1\right)}\right]\right\}\right) \cap \sigma^{\beta_{0}}\right] \\
& \subseteq \operatorname{pow}^{*}\left(\left\{{\overline{\alpha_{1}}}^{\left(r_{0}-1\right)}, \ldots,{\overline{\alpha_{\ell}}}^{\left(r_{0}-1\right)}\right\}\right) \cap{\overline{\beta_{0}}}^{\left(r_{0}-1\right)} \\
& \subseteq{\overline{\beta_{0}}}^{\left(r_{0}-1\right)} \\
& \subseteq \bigcup_{\beta \in T(A)} \bar{\beta}^{\left(r_{0}-1\right)} .
\end{aligned}
$$


The latter inclusion contradicts our earlier assumption $\Delta_{\beta_{0}}^{\left(r_{0}\right)} \neq \emptyset$, thereby showing that the place $\beta_{0}$ must be unblocked even in the present case. Thus Assertion $(\mathrm{B} .3)^{\left({ }_{0}\right)}$ is fully proved.

After the assignment statements have been executed, we have

$$
\bar{\pi}^{\left(r_{0}\right)}=\bar{\pi}^{\left(r_{0}-1\right)} \cup \Delta_{\pi}^{\left(r_{0}\right)}, \text { for each } \pi \in \Pi,
$$

and

$$
f^{\left(r_{0}\right)}=\left.f^{\left(r_{0}-1\right)} \cup \overline{f^{\left(r_{0}-1\right)}}\right|_{\text {pow } \omega^{*}\left(\left\{{\overline{\alpha_{1}}}^{\left(r_{0}-2\right)}, \ldots,{\overline{\alpha_{l}}}^{\left(r_{0}-1\right)}\right\}\right)} .
$$

To complete our analysis of the inductive step in the case in which $\left|\overline{\alpha_{i}}\left(r_{0}-1\right)\right|$ $<\rho$ for all $i=1, \ldots \ell$, we only need to verify Assertion $E^{\left(r_{0}\right)}$. This is done as follows.

Concerning $(\mathrm{E} .1)^{\left(r_{0}\right)}$, we begin by showing that $f^{\left(r_{0}\right)}$ is indeed a function, i.e. that for each $u$ in $\operatorname{dom}\left(f^{\left(r_{0}\right)}\right)$ there is exactly one pair $(u, t) \in f^{\left(r_{0}\right)}$ having $u$ as first element. As by induction $f^{\left(r_{0}-1\right)}$ and $\overline{f^{\left(r_{0}-1\right)}}$ are functions, it is enough to prove that for each $u \in \operatorname{dom}\left(f^{\left(r_{0}-1\right)}\right) \cap p o w^{*}\left(\left\{{\overline{\alpha_{1}}}^{\left(r_{0}-1\right)}, \ldots,{\overline{\alpha_{l}}}^{\left(r_{0}-1\right)}\right\}\right)$, $f^{\left(r_{0}-1\right)}(u)=f^{\left(r_{0}-1\right)}[u]$. But this follows plainly from Assertion $(\text { E.5 })^{\left(r_{0}-1\right)}$. Next we prove that $f^{\left(r_{0}\right)}$ is injective. As by induction and by Lemma 2.7 both $f^{\left(r_{0}-1\right)}$ and $\overline{f^{\left(r_{0}-1\right)}}$ are injective, it is enough to prove the following lemma.

LEMMA 4.2 Let $u+1 \in \operatorname{dom}\left(f^{\left(r_{0}-1\right)}\right)$ and $u_{2} \in$ pow $\left(\left\{\bar{\gamma}^{\left(r_{0}-1\right)}: \gamma \in \Gamma\right\}\right)$ such that $f^{\left(r_{0}-1\right)}\left(u_{1}\right)=\overline{f^{\left(r_{0}-1\right)}}\left(u_{2}\right)$, where $\Gamma$ is a $P$-node with $\left|\bar{\gamma}^{\left(r_{0}-1\right)}\right|<\rho$ for all $\gamma \in \Gamma$. Then $u_{1}=u_{2}$.

Proof. Let $u_{1}, u_{2}$ and $\Gamma$ be as in the above hypotheses. Then from Lemma 2.7 and Lemma 5.1 (iii) it follows that $f^{\left(r_{0}-1\right)}\left(u_{1}\right) \in p o w^{*}\left(\left\{\sigma^{\gamma}: \gamma \in \Gamma\right\}\right)$, which by $(E .8)^{\left(r_{0}-1\right)}$, by the disjointness of the sets $\sigma$ 's and Lemma 2.6(c) implies $u_{1} \in$ pow $^{*}\left(\left\{\widehat{\gamma}^{\left(r_{0}-1\right)}: \gamma \in \Gamma\right\}\right)$. Therefore $(E .2)^{\left(r_{0}-1\right)}$ and $(E .7)^{\left(r_{0}-1\right)}$ yield $f^{\left(r_{0}-1\right)}\left(u_{1}\right)=\overline{f^{\left(r_{0}-1\right)}}\left(u_{1}\right)=\overline{f^{\left(r_{0}-1\right)}}\left(u_{2}\right)$, which by the injectivity of $\overline{f^{\left(r_{0}-1\right)}}$ implies $u_{1}=u_{2}$, thus proving the lemma.

Having proved the preceding lemma, and thus established $(\mathrm{E} .1)^{\left(r_{0}\right)}$, we turn to $(\mathrm{E} .2)^{\left(r_{0}\right)}$. To prove that $(\mathrm{E} .2)^{\left(r_{0}\right)}$ is satisfied it is enough to observe that, by $(\mathrm{B} .2)^{\left(r_{0}-1\right)}$,

$$
\operatorname{pow}^{*}\left(\left\{{\overline{\alpha_{1}}}^{\left(r_{0}-1\right)}, \ldots, \overline{\alpha \ell}^{\left(r_{0}-1\right)}\right\}\right) \subseteq \bigcup_{\beta \in T(A)} \bar{\beta}^{\left(r_{0}-1\right)} \cup \bigcup_{\beta \in T(A)} \Delta_{\beta}^{\left(r_{0}\right)}=\bigcup_{\beta \in T(A)} \bar{\beta}^{\left(r_{0}\right)} .
$$

Next we prove that $(\text { E. } 3)^{\left(r_{0}\right)}$ holds. Let $\pi \in \Pi$. Since $f^{\left(r_{0}\right)}\left[\bar{\pi}^{\left(r_{0}\right)}\right]=$ $f^{\left(r_{0}-1\right)}\left[\bar{\pi}^{\left(r_{0}-1\right)}\right] \cup \overline{f^{\left(r_{0}-1\right)}}\left[\Delta_{\pi}^{\left(r_{0}\right)}\right]$, by inductive hypothesis we can limit ourselves 
to proving $\overline{f^{\left(r_{0}-1\right)}}\left[\Delta_{\pi}^{\left(r_{0}\right)}\right] \subseteq \sigma^{\pi}$. But this follows immediately from the very definition of $\Delta_{\pi}^{\left(r_{0}\right)}$. Since $\Delta_{\pi}^{\left(r_{0}\right)} \subseteq \operatorname{dom}\left(f^{\left(r_{0}\right)}\right)$, for all $\pi \in \Pi$, by induction we obtain $(\mathrm{E} .4)^{\left(r_{0}\right)}$.

Next we verify Assertion $(E .5)^{\left(r_{0}\right)}$. Let $\pi$ be a trapped place. From $(B .2)^{\left(r_{0}\right)}$ and the inductive hypothesis $(\mathrm{E} .5)^{\left(r_{0}-1\right)}$, we have $\bar{\pi}^{\left(r_{0}\right)} \subseteq \operatorname{dom}\left(f^{\left(r_{0}\right)}\right)$. Moreover $(\text { E.3 })^{\left(r_{0}\right)}$ implies $f^{\left(r_{0}\right)}\left[\bar{\pi}^{\left(r_{0}\right)}\right] \subseteq \sigma^{\pi}$. Therefore, as $f^{\left(r_{0}\right)}$ is injective we have $\left|\bar{\pi}^{\left(r_{0}\right)}\right| \leq\left|\sigma^{\pi}\right|$, which proves $(\mathrm{E} .5)^{\left(r_{0}\right)}$.

Concerning $(E .6)^{\left(r_{0}\right)}$, we need to prove that if $\alpha$ and $\beta$ are two distinct places of $\phi$, then $\bar{\alpha}^{\left(r_{0}\right)} \cap \bar{\beta}^{\left(r_{0}\right)}=0$. But $\bar{\alpha}^{\left(r_{0}\right)} \cap \bar{\beta}^{\left(r_{0}\right)}=\left(\bar{\alpha}^{\left(r_{0}-1\right)} \cap \Delta_{\beta}^{\left(r_{0}\right)}\right) \cup$ $\left(\bar{\beta}^{\left(r_{0}-1\right)} \cap \Delta_{\alpha}^{\left(r_{0}\right)}\right)$ therefore it is enough to show that $\bar{\gamma}^{\left(r_{0}-1\right)} \cap \Delta_{\delta}^{\left(r_{0}\right)}=\emptyset$, for any two distinct places $\gamma$ and $\delta$. Suppose by contradiction that there exists $u \in \bar{\gamma}^{\left(r_{0}-1\right)} \cap \Delta_{\delta}^{\left(r_{0}\right)}$. Then, by $(B .2)^{\left(r_{0}\right)}, u \in$ pow $^{*}\left(\left\{{\overline{\alpha_{1}}}^{\left(r_{0}-1\right)}, \ldots,{\overline{\alpha_{\ell}}}^{\left(r_{0}-1\right)}\right\}\right)$. In view of $(\text { E.9.b })^{\left(r_{0}-1\right)}$, this implies that $\Delta_{\delta}^{\left(r_{0}\right)} \Gamma^{\left(r_{0}-1\right)}=\emptyset$, thus completing the proof that Assertion (E.6) ${ }^{\left(r_{0}\right)}$ is valid.

Concerning $(\text { E. } 7)^{\left(r_{0}\right)}$, let $u \in$ pow $^{*}\left(\left\{\widehat{\gamma}^{\left(r_{0}\right)}: \gamma \in \Gamma\right\}\right) \cap \bigcup_{x \in \Pi} \bar{\pi}^{\left(r_{0}\right)}$, with $\Gamma$ a P-node such that $\left|\bar{\gamma}^{\left(r_{0}\right)}\right|<\rho$ for each $\gamma \in \Gamma$. Let $C_{r^{\prime}}$, with $r^{\prime} \leq r_{0}$, be the call to Distribute during whose execution the element $u$ is introduced into $\bigcup_{\pi \in \Pi} \bar{\pi}$. Notice that by the disjointness of the sets $\bar{\pi}^{\left(r^{\prime}-1\right)}, C_{r^{\prime}}$ must be the call Distribute( $\Gamma)$. As $\left|\bar{\gamma}^{\left(r^{\prime}-1\right)}\right|<\rho$ for each $\gamma \in \Gamma$, it follow's that $u \in$ pow* $\left(\left\{\bar{\gamma}^{\left(r^{\prime}-1\right)}: \gamma \in \Gamma\right\}\right) \subseteq \operatorname{dom}\left(\underline{\left.f^{\left(r^{\prime}-1\right)}\right)} \cap \operatorname{dom}\left(f^{\left(\tau^{\prime}\right)}\right) \subseteq \operatorname{dom}\left(\overline{f^{\left(r_{0}\right)}}\right) \cap \operatorname{dom}\left(f^{\left(r_{0}\right)}\right)\right.$, and also $f^{\left(r_{0}\right)}(u)=f^{\left(r^{\prime}\right)}(u)=\overline{f^{\left(r^{\prime}-1\right)}}[u]=f^{\left(r^{\prime}-1\right)}[u]=f^{\left(r_{0}\right)}[u]$, which proves Assertion (E.7) ${ }^{\left(r_{0}\right)}$.

Concerning $(\text { E. } 8)^{\left(r_{0}\right)}$, let $u \in$ pow $^{*}\left(\left\{\bar{\gamma}^{\left(r_{0}\right)}: \gamma \in \Gamma\right\}\right) \cap \operatorname{dom}\left(f^{\left(r_{0}\right)}\right)$, where $\Gamma$ is any P-node. From $(\mathrm{E} .2)^{\left(r_{0}\right)}$, we have $u \in \bar{\pi}^{\left(r_{0}\right)}$, for some $\pi \in \Pi$. Let $C_{r^{\prime}}$, with $\boldsymbol{r}^{\prime} \leq r_{0}$, be the call Distribute $(\Gamma)$ during whose execution the element $u$ is put into $\bigcup_{\pi \in \Pi} \bar{\pi}$. If $\left|\bar{\gamma}^{\left(r^{\prime}-1\right)}\right| \geq \rho$ for some $\gamma \in \Gamma$, then $f^{\left(r^{\prime}\right)}(u) \in \sigma^{\pi_{0}} \cap \operatorname{pow}^{*}\left(\left\{\sigma^{\gamma}\right.\right.$ : $\gamma \in \Gamma\}) \backslash\left(\sigma^{\Gamma}\right)^{\left(r^{\prime}\right)} \backslash \operatorname{range}\left(f^{\left(r^{\prime}-1\right)}\right)$. On the other hand, if $\left|\bar{\gamma}^{\left(r^{\prime}-1\right)}\right|<\rho$ for all $\gamma \in \Gamma$, then by Lemma 4.1(iii) $f^{\left(r^{\prime}\right)}(u)=\overline{f^{\left(r^{\prime}-1\right)}}(u) \in \operatorname{pow}^{*}\left(\left\{\sigma^{\gamma}: \gamma \in \Gamma\right\}\right)$. In any case we have $f^{\left(r_{0}\right)}(u)=f^{\left(r^{\prime}\right)}(u) \in \operatorname{pow}^{*}\left(\left\{\sigma^{\gamma}: \gamma \in \Gamma\right\}\right)$, which proves $(\mathrm{E} .8)^{\left(r_{0}\right)}$.

Finally, in order to complete our analysis of the inductive step, at least in the case in which $\left|\bar{\alpha}_{i}^{\left(r_{0}-1\right)}\right|<\rho$ for all $i=1, \ldots, l$, we only need to prove that Assertion $(\text { E.9 })^{\left(r_{0}\right)}$ is satisfied too. Let $t \in \operatorname{pow}^{*}\left(\left\{\overline{\gamma_{1}}\left(r_{0}\right), \ldots, \overline{\gamma_{h}}\left(r_{0}\right)\right\}\right) \cap \bar{\delta}^{\left(r_{0}\right)}$, for some places $\gamma_{1}, \ldots, \gamma_{h}, \delta$. Let $C_{r^{\prime}}$ be the call to the procedure Distribute during whose execution the element $t$ is introduced into the set $\bar{\delta}$. Suppose that $C_{r^{\prime}}$ is the call Distribute $\left(\beta_{1}, \ldots, \beta_{k}\right)$, with $\left\{\beta_{1}, \ldots, \beta_{k}\right\}$ a P-node. Therefore by inductive hypothesis $(\mathrm{B} .2)^{\left(r^{\prime}\right)}$ and $(\mathrm{C} .3)^{\left(r^{\prime}\right)} t \in \operatorname{pow}^{*}\left(\left\{{\overline{\beta_{1}}}^{\left(r^{\prime}-1\right)}, \ldots,{\overline{\beta_{k}}}^{\left(r^{\prime}-1\right)}\right\}\right) \subseteq$ 
$\operatorname{pow}^{*}\left(\left\{{\overline{\beta_{1}}}^{\left(r_{0}\right)}, \ldots,{\overline{\beta_{k}}}^{\left(r_{0}\right)}\right\}\right)$. The latter relationships imply $\left\{\beta_{1}, \ldots, \beta_{k}\right\}=\left\{\gamma_{1}\right.$, $\left.\ldots, \gamma_{h}\right\}$, since the sets ${\overline{\beta_{1}}}^{\left(r_{0}\right)}, \ldots,{\overline{\beta_{k}}}^{\left(r_{0}\right)},{\overline{\gamma_{1}}}^{\left(r_{0}\right)}, \ldots, \overline{\gamma h}^{\left(r_{0}\right)}$ are pairwise disjoint, if not identical (cf. Lemma 2.6(b)). Therefore by $(B .3)^{\left(r^{\prime}\right)}$ and $(\mathrm{C} .3)^{\left(r^{\prime}\right)}$ we have that $\left\{\gamma_{1}, \ldots, \gamma_{h}\right\}$ is a $P$-node having $\delta$ among its targets, thus proving $\left(\text { E.9.a) }{ }^{\left(r_{0}\right)} \text { and }(E .9 . b)^{\left(r_{0}\right)} \text {. Furthermore (E.9.c) }\right)^{\left(r_{0}\right)}$ follows immediately from the above discussion, so that Assertion (E.9) $)^{\left(r_{0}\right)}$ holds. This completes the analysis of our first case. The second, and last, case to be considered is the following.

Case: $\left|{\overline{\alpha_{0}}}^{\left(r_{0}-1\right)}\right| \geq \rho$ for some $j_{0} \in\{1, \ldots, \ell\}$. Let $\beta_{1}, \ldots, \beta_{g}, \tau_{1}, \ldots, \tau_{k}$, $\nu_{1}, \ldots, \nu_{h}$ be all the targets of the P-node $A$, where at the time the call $C_{r_{0}}$ is made the $\beta$ 's are the blocked targets, the $\tau$ 's are the unblocked trapped targets, and the $\nu$ 's are the unblocked untrapped targets of $\left\{\alpha_{1}, \ldots, \alpha_{\ell}\right\}$, respectively.

We have

$$
\begin{aligned}
& \Delta^{\prime\left(r_{0}\right)}=\operatorname{pow}^{*}\left(\left\{{\overline{\alpha_{1}}}^{\left(r_{0}-1\right)}, \ldots,{\overline{\alpha_{\ell}}}^{\left(r_{0}-1\right)}\right\}\right) \backslash \bigcup_{\beta \in T(A)} \bar{\beta}^{\left(r_{0}-1\right)}
\end{aligned}
$$

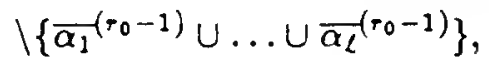

$$
\begin{aligned}
& \Delta^{\prime\left(r_{0}\right)}= \begin{cases}0 & \text { if } A \text { is not blocked just before } \\
\left\{\bar{\alpha}_{1}\left(r_{0}-1\right) \cup \ldots \cup{\overline{\alpha_{\ell}}}^{\left(r_{0}-1\right)}\right\} & \text { the call } C_{r_{0}} \text { is made } \\
\text { otherwise. }\end{cases} \\
& \Delta^{\left(r_{0}\right)}=\Delta^{\prime\left(r_{0}\right)} \cup \Delta^{\prime \prime\left(r_{0}\right)} \text {, } \\
& \left(\sigma^{A}\right)^{\left(r_{0}\right)}= \begin{cases}\emptyset & \text { if } A \text { is blocked just before the call } \\
\left\{\sigma^{\alpha_{1}} \cup \ldots \cup \sigma^{\alpha_{\ell}}\right\} & C_{r_{0}} \text { is made } \\
\text { otherwise. }\end{cases}
\end{aligned}
$$

In addition, for all unblocked targets $\tau_{1}, \ldots, \tau_{k}$ of $A$, we have

$$
\begin{aligned}
n_{j}^{\left(r_{0}\right)}= & \left|\sigma^{\tau_{j}} \cap \operatorname{pow}^{*}\left(\left\{\sigma^{\alpha_{1}}, \ldots, \sigma^{\alpha_{\ell}}\right\}\right) \backslash\left(\sigma^{A}\right)^{\left(r_{0}\right)}\right| \\
& -\left|\bar{\tau}_{j}^{\left(r_{0}-1\right)} \cap \operatorname{pow}^{*}\left(\left\{{\overline{\alpha_{1}}}^{\left(r_{0}-1\right)}, \ldots,{\overline{\alpha_{l}}}^{\left(r_{0}-1\right)}\right\}\right)\right| .
\end{aligned}
$$

We begin by proving that Assertion $\mathrm{C}^{\left(r_{0}\right)}$ is satisfied.

Concerning $(C .1)^{\left(r_{0}\right)}$, assume that ${\overline{\alpha_{1}}}^{\left(r_{0}-1\right)} \cup \ldots \cup{\overline{\alpha_{l}}}^{\left(r_{0}-1\right)} \in \bar{\beta}^{\left(r_{0}-1\right)}$, for some place $\beta_{0} \in T(A)$. Then the element $\overline{\alpha_{1}}\left(r_{0}\right) \cup \ldots \cup \overline{\alpha_{\ell}}\left(r_{0}\right)$ has been introduced into the set $\bar{\beta}_{0}$ during the execution of a prior call $C_{r^{\prime}}$, Distribute $\left(\alpha_{1}, \ldots \alpha_{l}\right)$, and furthermore $\overline{\alpha_{1}}\left(^{\prime}-1\right) \cup \ldots \cup \overline{\alpha_{\ell}}{ }^{\left(r^{\prime}-1\right)}={\overline{\alpha_{1}}}^{\left(r_{0}-1\right)} \cup \ldots \cup \overline{\alpha_{\ell}}\left(r_{0}-1\right)$. As ${\overline{\alpha_{1}}}^{\left(r^{\prime}-1\right)} \cup$ $\ldots \cup \bar{\alpha}_{\ell}\left(r^{\prime}-1\right) \in \Delta^{\left(r^{\prime}\right)}$, the P-node $A$ must have been blocked when the call $C_{r^{\prime}}$ was made, preventing it from being processed again by procedure Distribute. 
But this is a contradiction, since the call $C_{r_{0}}$ has as argument the P-node $A$. Therefore

$$
{\overline{\alpha_{1}}}^{\left(r_{0}-1\right)} \cup \ldots \cup{\overline{\alpha_{\ell}}}^{\left(r_{0}-1\right)} \notin \bigcup_{\beta \in T(A)} \bar{\beta}^{\left(r_{0}-1\right)},
$$

proving $(\mathrm{C} .1)^{\left(\tau_{0}\right)}$.

Concerning $(\mathrm{C} .2)^{\left(r_{0}\right)}$, suppose by contradiction that $\sigma^{\alpha_{1}} \cup \ldots \cup \sigma^{\alpha_{\ell}} \in$ range $\left(f^{\left(r_{0}-1\right)}\right)$, and let $u \in \operatorname{dom}\left(f^{\left(r_{0}-1\right)}\right)$ such that $\left.f^{\left(r_{0}-1\right)}(u)=\sigma^{a_{1}}\right\rfloor \ldots \cup \sigma^{\alpha_{\ell}}$. Notice that the inductive hypothesis $(\text { E. } 8)^{\left(r_{0}-1\right)}$ implies $u \in$ pow $^{*}\left(\left\{\bar{a}_{:}^{\left(r_{0}-1\right)}, \ldots\right.\right.$, $\left.\left.\overline{\alpha_{\ell}}\left(r_{0}-1\right)\right\}\right)$, so that the element $u$ must have been introduced during a prior call $C_{r^{\prime}}$, Distribute $\left(\alpha_{1}, \ldots, \alpha_{\ell}\right)$. If $\left|\alpha_{i}^{\left(r^{\prime}-1\right)}\right|<\rho$ for all $i=1, \ldots \ell$, then $\sigma^{\alpha_{1}} \cup \ldots U$ $\sigma^{\alpha_{\ell}}=f^{\left(r^{\prime}\right)}(u)=f^{\left(r^{\prime}-1\right)}[u] \subseteq f^{\left(r^{\prime}-1\right)}\left[\alpha_{1}\left(r^{\prime}-1\right)\right] \cup \ldots \cup f^{\left(r^{\prime}-1\right)}\left[\overline{\alpha_{\ell}}\left(r^{\prime}-1\right)\right.$. and since by Lemma $4.1\left(\right.$ ii) $f^{\left(r^{\prime}-1\right)}\left[\overline{\alpha_{i}}\left(r^{\prime}-1\right)\right] \subseteq \sigma^{\alpha_{i}}$, for $i=1, \ldots, \ell$, then $\left.f^{\left(r^{\prime}-1 ; \overline{\alpha_{i}}\left(r^{\prime}-1\right)\right.}\right]=$ $\sigma^{\alpha_{i}}, i=1, \ldots, \ell$. In view of the injectivity of $f^{\left(r^{\prime}-1\right)}$, the latter equaities imply $\left|\sigma^{\alpha_{i}}\right|=\left|\overline{\alpha_{i}}\left(r^{\prime}-1\right)\right|$, i.e. the places $\alpha_{i}$ are trapped, for $i=1, \ldots, \ell$. But then by inductive hypothesis $(E .5)^{\left(r_{0}-1\right)}$, we should have $\left|\bar{\alpha}_{i}\left(r^{\prime}-1\right)\right|<\rho$. for all $i=$ $1, \ldots, \ell$, contradicting our assumption $\left|\overline{\alpha_{j_{0}}}\left(r_{0}-1\right)\right| \geq \rho$ for some $j_{0} \in\{1, \ldots, \ell\}$. This contradition shows that $\left|\bar{\alpha}_{j}\left(r^{\prime}-1\right)\right| \geq \rho$ for some $j \in\{1, \ldots, \ell\}$. But then, an argument similar to the one given for the proof of Assertion $(C .1)^{\left(r_{0}\right)}$ allows us to conclude that $(\mathrm{C} .2)^{\left(r_{0}\right)}$ is also satisfied.

Next we show that Assertions $(\mathrm{C} .3)^{\left(r_{0}\right)}$ and $(\mathrm{C} .4)^{\left(r_{0}\right)}$ hold too. If the P-node $\left\{\alpha_{1}, \ldots, \alpha_{\ell}\right\}$ has never been processed by Distribute prior to the call $C_{r_{0}}$, we put $r^{\prime}=1$, otherwise we let $C_{\tau^{\prime}}$ denote the latest call Distribute $\left(\alpha_{1}, \ldots, \alpha_{\ell}\right)$, with $r^{\prime}<r_{0}$. We will distinguish two subcases, according to whether the set $\bigcup_{\alpha \in A}\left(\bar{\alpha}^{\left(r_{0}-1\right)} \backslash \bar{\alpha}^{\left(r^{2}-1\right)}\right)$ is empty or not.

Subcase: $\bigcup_{\alpha \in A}\left(\bar{\alpha}^{\left(r_{0}-1\right)} \backslash \bar{\alpha}^{\left(r^{\prime}-1\right)}\right)=\emptyset$. Notice that in this case $r^{\prime}>1$, and $\bar{\alpha}^{\left(r_{0}-1\right)}=\bar{\alpha}^{\left(r^{\prime}-1\right)}$, for all $\alpha \in A$. Therefore after the execution of $C_{r^{\prime}}$, the P-node $A$ is marked as 'visited', preventing it from being further processed from the Propagation Phase. Thus the call $C_{r_{0}}$ must have been made from the Blocking Phase, and in particular the P-node $A$ must be marked 'blocked' when the call $C_{r_{0}}$ is made. Therefore, $\Delta^{\prime \prime\left(r_{0}\right)}=\left\{\bar{\alpha}_{1}^{\left(r_{0}-1\right)} \cup \ldots \cup \bar{\alpha}^{\left(r_{0}-1\right)}\right\}$, $\left(\sigma^{A}\right)^{\left(r_{0}\right)}=\emptyset$, and, by the inductive hypothesis $C^{\left(r^{\prime}\right)}, \Delta^{\prime\left(r_{0}\right)}=0$ and $\Delta^{\left(r_{0}\right)}=$ $\Delta^{\prime \prime\left(r_{0}\right)}$. Assertion (C.3.b) $)^{\left(r_{0}\right)}$ is vacuously true. As regards (C.3.c) $)^{\left(r_{0}\right)}$, it is sufficient to notice that as $\pi^{A}>\alpha$, for all $\alpha \in A$ (cf. Lemma 3.12), then Lemma 3.13(d) assures us that the place $\pi^{A}$ is unblocked during the execution fo the call $C_{r_{0}}$ (we recall that $\pi^{A}$ denotes the principal target of the P-node $A$ ). In order to prove $(\mathrm{C} .3 . \mathrm{a})^{\left(r_{0}\right)}$, all we have to show is that $n_{j}^{\left(r_{0}\right)} \leq 1, j=1, \ldots, k$, where the equality holds if and only if $\tau_{j}$ coincides with the place $\pi^{1}$. We have

$$
n_{j}^{\left(\tau^{\prime}\right)}=\mid \sigma^{\tau_{j}} \cap p o w^{*}\left(\left\{\sigma^{\alpha_{1}}, \ldots, \sigma^{\alpha_{\ell}}\right\}\right) \backslash\left\{\sigma^{\alpha_{1}} \cup \ldots \cup \sigma^{\alpha_{\ell}}\right\}
$$




$$
-\left|{\overline{\tau_{j}}}^{\left(r^{\prime}-1\right)} \cap \operatorname{pow}^{*}\left(\left\{{\overline{\alpha_{1}}}^{\left(r^{\prime}-1\right)}, \ldots,{\overline{\alpha_{\ell}}}^{\left(r^{\prime}-1\right)}\right\}\right)\right|
$$

and $\overline{\tau_{j}}\left(r^{\prime}\right)=\overline{\tau_{j}}\left(r^{\prime}-1\right) \cup \Delta_{\tau_{j}}^{\left(r^{\prime}\right)}$. Therefore, by inductive hypothesis,

$$
\begin{aligned}
& \left|\sigma^{\tau_{1}} \cap \operatorname{pow} w^{*}\left(\left\{\sigma^{\alpha_{1}}, \ldots, \sigma^{\alpha_{\ell}}\right\}\right)\right|-n_{j}^{\left(r_{0}\right)} \\
& =\left|{\overline{\tau_{j}}}^{\left(r_{0}-1\right)} \cap \operatorname{pow}^{*}\left(\left\{{\overline{\alpha_{1}}}^{\left(r_{0}-1\right)}, \ldots,{\overline{\alpha_{\ell}}}^{\left(r_{0}-1\right)}\right\}\right)\right| \\
& =\mid{\overline{\tau_{j}}}^{\left(r^{\prime}\right)} \cap \text { pow }\left(\left\{{\overline{\alpha_{1}}}^{\left(r^{\prime}-1\right)}, \ldots,{\overline{\alpha_{\ell}}}^{\left(r^{\prime}-1\right)}\right\}\right) \mid \\
& =\mid\left(\overline{\tau j}^{\left(r^{\prime}-1\right)} \cup \Delta_{\tau_{j}}^{\left(r^{\prime}\right)}\right) \cap \text { pow }\left(\left\{{\overline{\alpha_{1}}}^{\left(r^{\prime}-1\right)}, \ldots, \bar{\alpha}_{l}^{\left(r^{\prime}-1\right)}\right\}\right) \mid \\
& =\mid{\overline{\tau_{j}}}^{\left(r^{\prime}-1\right)} \cap \text { pow }^{*}\left(\left\{\overline{\alpha_{1}}\left(r^{\prime}-1\right), \ldots, \overline{\alpha_{\ell}}\left(r^{\prime}-1\right)\right\}\right)|+| \Delta_{\tau_{j}}^{\left(r^{\prime}\right)} \mid \\
& =\left|{\overline{\tau_{j}}}^{\left(r^{\prime}-1\right)} \cap \operatorname{pow}^{*}\left(\left\{{\overline{\alpha_{1}}}^{\left(r^{\prime}-1\right)}, \ldots, \overline{\alpha_{\ell}}{ }^{\left(r^{\prime}-1\right)}\right\}\right)\right|+n_{j}^{\left(r^{\prime}\right)} \\
& =\left|\sigma^{\tau_{1}} \cap \operatorname{pow}^{*}\left(\left\{\sigma^{\alpha_{1}}, \ldots, \sigma^{\alpha_{\ell}}\right\}\right) \backslash\left\{\sigma^{\alpha_{1}} \cup \ldots \cup \sigma^{\alpha_{\ell}}\right\}\right|,
\end{aligned}
$$

so that

$$
n_{j}^{\left(r_{0}\right)}=\left\{\begin{array}{cc}
1 & \text { if } \tau_{j} \equiv \pi^{A} \\
0 & \text { otherwise. }
\end{array}\right.
$$

concluding the proof of (C.3.a $)^{\left(r_{0}\right)}$ and in turn of Assertion (C.3 $)^{\left(r_{0}\right)}$, at least in the present case in which $\bigcup_{\alpha \in A}\left(\bar{\alpha}^{\left(r_{0}-1\right)} \backslash \bar{\alpha}^{\left(r^{\prime}-1\right)}\right)=\emptyset$.

Subcase: $\bigcup_{\alpha \in A}\left(\bar{\alpha}^{\left(r_{0}-1\right)} \backslash \bar{\alpha}^{\left(r^{\prime}-1\right)}\right)=\emptyset$. Notice that $n_{j}^{\left(r_{0}\right)} \leq\left|\sigma^{\tau_{1}}\right|<\rho$, for each trapped target $\tau_{j}$ of $A$. Hence, by Lemma 3.9(b) and since by Lemma 3.6(e) each $\mathrm{P}$-node can have at most $n-1$ targets, to prove $(\mathrm{C} .3)^{\left(r_{0}\right)}$ it is enough to show that $\left|\Delta^{\left(r_{0}\right)}\right| \geq \rho(n-1)$.

CLAIM. For each $t_{0} \in \bigcup_{\alpha \in A}\left(\bar{\alpha}^{\left(r_{0}-1\right)} \backslash \bar{\alpha}^{\left(r^{\prime}-1\right)}\right)$ we have:

(I) $\operatorname{pow}^{*}\left(\left\{\left\{t_{0}\right\},{\overline{\alpha_{1}}}^{\left(r_{0}-1\right)}, \ldots,{\overline{\alpha_{\ell}}}^{\left(r_{0}-1\right)}\right\}\right) \subseteq \Delta^{\left(r_{0}\right)} \cup\left\{{\overline{\alpha_{1}}}^{\left(r_{0}-1\right)} \cup \ldots \cup{\overline{\alpha_{\ell}}}^{\left(r_{0}-1\right)}\right\}$

(II) $\left|p o w *\left(\left\{\left\{t_{0}\right\},{\overline{\alpha_{1}}}^{\left(r_{0}-1\right)}, \ldots,{\overline{\alpha_{\ell}}}^{\left(r_{0}-1\right)}\right\}\right)\right|>\rho(n-1)$.

Proof. As $\left\{t_{0}\right\} \subseteq \bigcup_{\alpha \in A} \bar{\alpha}^{\left(r_{0}-1\right)}$, we have clearly

$$
\operatorname{pow}^{*}\left(\left\{\left\{t_{0}\right\},{\overline{\alpha_{1}}}^{\left(r_{0}-1\right)}, \ldots,{\overline{\alpha_{l}}}^{\left(r_{0}-1\right)}\right\}\right) \subseteq \operatorname{pow}^{*}\left(\left\{{\overline{\alpha_{1}}}^{\left(r_{0}-1\right)}, \ldots,{\overline{\alpha_{\ell}}}^{\left(r_{0}-1\right)}\right\}\right) .
$$

Therefore, to prove (I) it suffices to show that

$$
\operatorname{pow}^{*}\left(\left\{\left\{t_{0}\right\},{\overline{\alpha_{1}}}^{\left(r_{0}-1\right)}, \ldots,{\overline{\alpha_{\ell}}}^{\left(r_{0}-1\right)}\right\}\right) \cap \bigcup_{\beta \in T(A)} \bar{\beta}^{\left(r_{0}-1\right)}=\emptyset .
$$


We do this as follows. Let $u_{0} \in$ pow $\left(\left\{\left\{t_{0}\right\},{\overline{\alpha_{1}}}^{\left(r_{0}-1\right)}, \ldots,{\overline{a_{l}}}^{\left(r_{0}-1\right)}\right\}\right) \cap \bar{\beta}^{\left(r_{0}-1\right)}$, for some $\beta_{0} \in T(A)$. By inductive hypothesis $(E .9)^{\left(r_{0}-1\right)}$, the element $u_{0}$ has been introduced $\bar{\beta}$ during the execution of a call $C_{\mathbf{r}}$, Distribute $\left(a_{1}, \ldots, \alpha_{\ell}\right)$, with $r^{\prime \prime} \leq r^{\prime}$. In particular, $t_{0} \in \bigcup_{\alpha \in A} \bar{\alpha}^{\left(r^{\prime}-1\right)} \subseteq \bigcup_{\alpha \in A} \bar{\alpha}^{\left(r^{\prime}-1\right)}$, contradicting the fact that $t_{0} \notin \bigcup_{\alpha \in A} \bar{\alpha}^{\left(r^{\prime}-1\right)}$, and thereby completing the proof of (I).

To prove (II) we just observe that as $\left|\overline{\alpha_{j_{0}}}\left(r_{0}-1\right)\right| \geq \rho$ for some $j_{0} \in\{1, \ldots \ell\}$, then obviously

$$
\left|p o w^{*}\left(\left\{\left\{t_{0}\right\},{\overline{\alpha_{1}}}^{\left(r_{0}-1\right)}, \ldots,{\overline{\alpha_{\ell}}}^{\left(r_{0}-1\right)}\right\}\right)\right| \geq \min \left(2^{\rho}-1,2^{\rho-1}-1\right)=2^{\rho-1}-1 .
$$

Then (II) follows plainly from the assumption $2^{\rho-1}-1>\rho(n-1)$.

Thus the Claim is fully established.

The above Claim obviously implies that $\left|\Delta^{\left(r_{0}\right)}\right| \geq \rho(n-1)$, which concludes the proof of Assertion (C.3) ${ }^{\left(r_{0}\right)}$ even in the case in which $\bigcup_{\alpha \in A}\left(\bar{\alpha}^{\left(r_{0}-1\right)}\right)$ $\left.\bar{\alpha}^{\left(\mathbf{r}^{\prime}-1\right)}\right) \neq \emptyset$.

Finally we show that Assertion $(\mathrm{C} .4)^{\left(r_{0}\right)}$ is satisfied too. It is enough to prove

$$
\begin{aligned}
& \left|\sigma^{\tau_{1}} \cap \operatorname{pow}^{*}\left(\left\{\sigma^{\alpha_{1}}, \ldots, \sigma^{\alpha_{\ell}}\right\}\right) \cap \operatorname{range}\left(f^{\left(r_{0}-1\right)}\right) \backslash\left(\sigma^{A}\right)^{\left(r_{c}\right)}\right| \\
= & \left|{\overline{\tau_{j}}}^{\left(r_{0}-1\right)} \cap \operatorname{pow}^{*}\left(\left\{{\overline{\alpha_{1}}}^{\left(r_{0}-1\right)}, \ldots,{\overline{\alpha_{l}}}^{\left(r_{0}-1\right)}\right\}\right)\right|,
\end{aligned}
$$

for each $j=1, \ldots, k$. As $\tau_{j}$ is trapped, by inductive hypothesis $(\text { E.4 })^{\left(r_{0}-1\right)}$ and $(\mathrm{E} .5)^{\left(r_{0}-1\right)},{\overline{\tau_{j}}}^{\left(r_{0}-1\right)} \subseteq \operatorname{dom}\left(f^{\left(r_{0}-1\right)}\right)$. Also from $(\mathrm{C} .2)^{\left(r_{0}\right)},\left(\sigma^{A}\right)^{\left(r_{0}\right)} \cap$ range $\left(f^{(-0-1)}\right)=\emptyset$. Therefore we can limit ourselves to proring that

$$
\begin{aligned}
& f^{\left(r_{0}-1\right)}\left[{\overline{\tau_{j}}}^{\left(r_{0}-10\right.} \cap \operatorname{pow}^{*}\left(\left\{{\overline{\alpha_{1}}}^{\left(r_{0}-1\right)}, \ldots,{\overline{\alpha_{l}}}^{\left(r_{0}-1\right)}\right\}\right)\right] \\
= & \sigma^{\tau_{1}} \cap p o w^{*}\left(\left\{\sigma^{\alpha_{1}}, \ldots, \sigma^{\alpha_{l}}\right\}\right) \cap \operatorname{range}\left(f^{\left(r_{0}-1\right)}\right) .
\end{aligned}
$$

We do this as follows. Let $u \in \overline{\tau_{j}}\left(r_{0}-1\right) \cap p o w^{*}\left(\left\{{\overline{\alpha_{1}}}^{\left(r_{0}-1\right)}, \ldots, \overline{\alpha_{\iota}}\left(r_{0}-1\right)\right\}\right)$. Then by inductive hypotheses $(\text { E.3 })^{\left(r_{0}-1\right)^{\prime}}-(E .5)^{\left(r_{0}-1\right)}$ and $(E .8)^{\left(r_{0}-1\right)}$, we have $f^{\left(r_{0}-1\right)}(u) \in$ $\sigma^{\tau}, \cap \operatorname{pow}^{*}\left(\left\{\sigma^{\alpha_{1}}, \ldots, \sigma^{\alpha_{l}}\right\}\right) \cap \operatorname{range}\left(f^{\left(r_{0}-1\right)}\right)$. To prove the converse inclusion, let

$$
t \in \sigma^{\tau_{j}} \cap \text { pow }\left(\left\{\sigma^{\alpha_{1}}, \ldots, \sigma^{\alpha_{\ell}}\right\}\right) \cap \operatorname{range}\left(f^{\left(r_{0}-1\right)}\right) \text {. }
$$

Let $u \in \operatorname{dom}\left(f^{\left(r_{0}-1\right)}\right)$ such that $f^{\left(r_{0}-1\right)}(u)=t$. From $(\text { E. } 3)^{\left(r_{0}-1\right)},(\text { E. } 8)^{\left(r_{0}-1\right)}$,

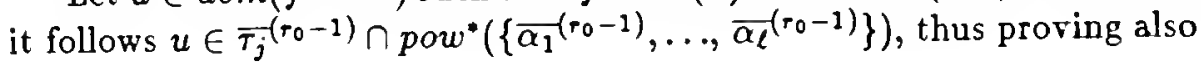

$$
\subseteq \begin{gathered}
\sigma^{\tau_{3}} \cap \operatorname{pow} w^{*}\left(\left\{\sigma^{\alpha_{1}}, \ldots, \sigma^{\alpha_{l}}\right\}\right) \cap \operatorname{range}\left(f^{\left(r_{0}-1\right)}\right) \\
\subseteq f^{\left(r_{0}-1\right)}\left[{\overline{\tau_{j}}}^{\left(r_{0}-1\right)} \cap \operatorname{pow}^{*}\left(\left\{{\overline{\alpha_{1}}}^{\left(r_{0}-1\right)}, \ldots,{\overline{\alpha_{l}}}^{\left(r_{0}-1\right)}\right\}\right)\right] .
\end{gathered}
$$


This proves the validity of Assertion (C.4 $)^{\left(r_{0}\right)}$, and in turn completes the demonstration that Assertion $C^{\left(r_{0}\right)}$ is satisfied.

After the execution of the assignment statements in $C_{r_{0}}$, we have

$$
\bar{\pi}^{\left(r_{0}\right)}=\bar{\pi}^{\left(r_{0}-1\right)} \cup \Delta_{\pi}^{\left(r_{0}\right)}, \text { for each } \pi \in \Pi,
$$

and

$$
f^{\left(r_{0}\right)}=f^{\left(r_{0}-1\right)} \cup f_{\tau_{1}}^{\left(r_{0}\right)} \cup \ldots \cup f_{\tau_{k}}^{\left(r_{0}\right)},
$$

where $f_{\tau_{j}}^{\left(r_{0}\right)}$ is a one-one correspondence between $\Delta_{\tau_{j}}^{\left(r_{0}\right)}$ and the set $\sigma^{\tau_{j}} \cap$ pow $\left(\left\{\sigma^{\alpha_{1}}, \ldots, \sigma^{\alpha_{\ell}}\right\}\right) \backslash\left(\sigma^{A}\right)^{\left(r_{0}\right)} \backslash \operatorname{range}\left(f^{\left(r_{0}-1\right)}\right), j=1, \ldots, k$.

To prove that Assertion $\mathrm{D}^{\left(r_{0}\right)}$ holds, we need Assertion (E.9) $)^{\left(r_{0}\right)}$, which can be proved exactly in the same way as in the previous case in which $\left|\bar{\alpha}_{i}^{\left(r_{0}-1\right)}\right|<\rho$ for all $i=1, \ldots, \ell$.

From $(\mathrm{E} .9)^{\left(r_{0}\right)}$ and $(\mathrm{C} .3)^{\left(r_{0}\right)}$ we have

$$
\begin{aligned}
& \left|{\overline{\tau_{j}}}^{\left(r_{0}\right)} \cap \operatorname{pow}^{*}\left(\left\{{\overline{\alpha_{1}}}^{\left(r_{0}\right)}, \ldots, \overline{\alpha_{\ell}}{ }^{\left(r_{0}\right)}\right\}\right)\right| \\
= & \left|\left({\overline{\tau_{j}}}^{\left(r^{\prime}-1\right)} \cup \Delta_{\tau_{j}}^{\left(r_{0}\right)}\right) \cap \operatorname{pow}^{*}\left(\left\{{\overline{\alpha_{1}}}^{\left(r_{0}-1\right)}, \ldots,{\overline{\alpha_{\ell}}}^{\left(r_{0}-1\right)}\right\}\right)\right| \\
= & \left|{\overline{\tau_{j}}}^{\left(r_{0}-1\right)} \cap \operatorname{pow}^{*}\left(\left\{{\overline{\alpha_{1}}}^{\left(r_{0}-1\right)}, \ldots,{\overline{\alpha_{\ell}}}^{\left(r_{0}-1\right)}\right\}\right)\right|+\left|\Delta_{\tau_{j}}^{\left(r_{0}\right)}\right| \\
= & \left|\sigma^{\tau_{j}} \cap \operatorname{pow}^{*}\left(\left\{\sigma^{\alpha_{1}}, \ldots, \sigma^{\alpha_{\ell}}\right\}\right) \backslash\left(\sigma^{A}\right)^{\left(r_{0}\right)}\right|
\end{aligned}
$$

for each $j=1, \ldots, k$, which proves that Assertion $\mathrm{D}^{\left(r_{0}\right)}$ is satisfied.

To complete the proof of the Main Inductive Lemma, we only need to show that in the present case in which $\left|{\overline{\alpha_{0}}}^{\left(r_{0}-1\right)}\right| \geq \rho$ for some $j_{0} \in\{1, \ldots, \ell\}$, Assertion $E^{\left(r_{0}\right)}$ is satisfied.

As regards $(E .1)^{\left(r_{0}\right)}$, it is enough to observe that $\left\{\operatorname{dom}\left(f^{\left(r_{0}-1\right)}\right)\right\}$ $\cup\left\{\operatorname{dom}\left(f_{\tau_{j}}^{\left(r_{0}\right)}\right): j=1, \ldots, k\right\}$ and $\left\{\operatorname{range}\left(f^{\left(r_{0}-1\right)}\right)\right\} \cup\left\{\operatorname{range}\left(f_{\tau_{0}}^{\left(r_{0}\right)}\right): j=\right.$ $1, \ldots, k\}$ are both families of pairwise disjoint sets, and that $f^{\left(r_{0}-1\right)}$, $f_{\tau_{1}}^{\left(r_{0}\right)}, \ldots, f_{\tau_{k}}^{\left(\tau_{0}\right)}$ are all 1-1 functions.

Concerning $(\text { E.2 })^{\left(r_{0}\right)}$, it suffices to notice that $\operatorname{dom}\left(f_{\tau_{j}}^{\left(r_{0}\right)}\right)=\Delta_{\tau_{j}}^{\left(r_{0}\right)} \subseteq \tau_{j}^{\left(r_{0}\right)}$, for all $j=1, \ldots, k$. Moreover, as $f_{\tau_{j}}^{\left(r_{0}\right)}\left[\Delta_{\tau_{j}}^{\left(r_{0}\right)}\right] \subseteq \sigma^{\tau_{j}}$, Assertion (E.3)( $\left.r_{0}\right)$ follows at once.

Next, let $\pi$ be a place of $\phi$ such that $\left|\bar{\pi}^{\left(r_{0}\right)}\right|<\rho$. Then $\left|\Delta_{\pi}^{\left(r_{0}\right)}\right|<\rho$, which easily implies $\Delta_{\pi}^{\left(r_{0}\right)} \subseteq \operatorname{dom}\left(f^{\left(r_{0}\right)}\right)$. Therefore by inductive hypothesis $(\mathrm{E} .4)^{\left(r_{0}-1\right)}$ we obtain (E.4) $)^{\left(r_{0}\right)}$.

Concerning (E.5) ${ }^{\left(r_{0}\right)}$, let $\pi$ be a trapped place. If $\pi$ is not a target of the P-node $A$, then $\bar{\pi}^{\left(r_{0}\right)}=\bar{\pi}^{\left(r_{0}-1\right)}$ and the inductive hypothesis $(\text { E.5 })^{\left(r_{0}-1\right)}$ implies (E.5) $\left(r_{0}\right)$. On the other hand, if $\pi$ is a target of the P-node $A$, then 
$\bar{\pi}^{\left(r_{0}\right)}=\bar{\pi}^{\left(r_{0}-1\right)} \cup \Delta_{\pi}^{\left(r_{0}\right)}$, with $\pi^{\left(r_{0}-1\right)} \subseteq \operatorname{dom}\left(f^{\left(r_{0}-1\right)}\right)\left(\right.$ cf. $\left.(\text { E.5 })^{\left(r_{0}-1\right)}\right)$ and $\Delta_{\pi}^{\left(r_{0}\right)} \subseteq$ $\operatorname{dom}\left(f_{\pi}^{\left(r_{0}\right)}\right) \subseteq \operatorname{dom}\left(f^{\left(r_{0}\right)}\right)$. Therefore, $\bar{\pi}^{\left(r_{0}\right)} \subseteq \operatorname{dom}\left(f^{\left(r_{0}\right)}\right)$, so that the injectivity of $f^{\left(r_{0}\right)}$ combined with $(\text { E. } 3)^{\left(r_{0}\right)}$ gives $\left|\bar{\pi}^{\left(r_{0}\right)}\right| \leq\left|\sigma^{\pi}\right|$, which proves $(\text { E. } 5)^{\left(r_{0}\right)}$.

As regards $(\mathrm{E} .6)^{\left(r_{0}\right)}$, let $\alpha$ and $\beta$ be any two distinct places of $\phi$. By inductive hypothesis, $\bar{\alpha}^{\left(r_{0}\right)} \cap \bar{\beta}^{\left(r_{0}\right)}=\left(\bar{\alpha}^{\left(r_{0}-1\right)} \cap \Delta_{\beta}^{\left(r_{0}\right)}\right) \cup\left(\bar{\beta}^{\left(r_{0}-1\right)} \cap \Delta_{a}^{\left(r_{0}\right)}\right)$, so that in order to prove that $\bar{\alpha}^{\left(r_{0}\right)} \cap \bar{\beta}^{\left(r_{0}\right)}=\emptyset$, it is enough to show that $\bar{\gamma}^{\left(r_{0}-1\right)} \cap \Delta_{\delta}^{\left(r_{0}\right)}=\emptyset$, for any two distinct places $\gamma$ and $\delta$. Suppose by contradiction that there exists $u \in \bar{\gamma}^{\left(r_{0}-1\right)} \cap \Delta_{\delta}^{\left(r_{0}\right)}$. Then, by $(\text { C.3 })^{\left(r_{0}\right)}, u \in$ pow $^{*}\left(\left\{\overline{\alpha_{1}}\left(r_{0}-1\right), \ldots, \bar{\alpha}_{\ell}^{\left(r_{0}-1\right)}\right\}\right)$, which by $(\text { E.9.b })^{\left(r_{0}-1\right)}$ implies that $\Delta_{\delta}^{\left(r_{0}\right)} \cap \bar{\gamma}^{\left(r_{0}-1\right)}=\emptyset$. But this contradicts our assumption $\bar{\gamma}^{\left(r_{0}-1\right)} \cap \Delta_{\delta}^{\left(r_{0}\right)} \neq \emptyset$, and consequently proves Assertion $(E .6)^{\left(r_{0}\right)}$.

Finally, concerning Assertions (E.7) ${ }^{\left(r_{0}\right)}$ and $(E .8)^{\left(r_{0}\right)}$, we notice that the proofs given in the analysis of the preceding case in which $\left|\bar{\alpha}_{j}^{-\left(r_{0}-1\right)}\right|<\rho$ for all $i=1, \ldots, \ell$ can be repeated word for word in the present case too. Also, we already observed that the same is true for Assertion (E. 9$)^{\left(r_{0}\right)}$.

This completes the analysis of the inductive step in the case in which $\left|{\overline{\alpha_{0}}}^{\left(r_{0}-1\right)}\right| \geq \rho$ for some $j_{0} \in\{1, \ldots, \ell\}$, thereby concluding the proof of the Main Inductive Lemma.

The following section will show that the sequence of calls $C_{1}, C_{2}, \ldots$, in $K$ is finite and that in fact the computation $K$ terminates.

\section{TERMINATION PROOF}

Again, we denote by $K$ an execution of the I.V.A.A. and by $C_{1}, C_{2}, C_{3}, \ldots$ the sequence of calls to the procedure Distribute arranged in the order in which they occur during the computation $K$. Also, we use the notation $\bar{\pi}^{(r)}, \Delta_{\pi}^{(r)}$, $f^{(r)}$, Assertion $\mathrm{X}^{(\boldsymbol{r})}$ with the same meaning as in the preceding sections.

In this section we prove the following lemma, which has been already stated in Section 3.

LEMMA 3.15 (Termination) The number of calls $C_{1}, C_{2}, C_{3}, \ldots$ in the computation $\mathrm{K}$ is bounded by $(\rho-2)(n-1) 2^{n-2}+3 \cdot 2^{n-1}-2$, where $n=|\Pi|$, and $\rho$ is the constant which appears in condition $\left(C_{4}\right)$ of Theorem 3.1. Moreover, when the last call to the procedure Distribute is made, all places $\pi \in \Pi$ are blocked.

Proof. We begin by establishing an upper bound on the number of calls $C_{1}, C_{2}, C_{3}, \ldots$ made during the computation $K$. Let $A=\left\{\alpha_{1}, \ldots \alpha_{\ell}\right\}$ be a 
nonempty $\mathrm{P}$-node, and let $C_{r_{1}}, C_{r_{2}}, C_{r_{3}}, \ldots$, with $1 \leq r_{1}<r_{2}<r_{3}<\ldots$, be the subsequence of $C_{1}, C_{2}, C_{3}, \ldots$ consisting of all the calls Distribute $\left(\alpha_{1}, \ldots, \alpha_{\ell}\right)$ made from the Propagation Phase and such that $\left|{\overline{\alpha_{i}}}^{(r,-1)}\right|<\rho$, for all $i=$ $1, \ldots, \ell$ and $j=1,2,3, \ldots$ It follows by $\left.(\text { B. } 2)^{(r,}\right)$ that for each $j=1,2,3, \ldots$

$$
\bigcup_{\beta \in T(A)} \bar{\beta}^{\left(r_{j+1}-1\right)} \supseteq \bigcup_{\beta \in T(A)} \bar{\beta}^{\left(r_{j}\right)} \supseteq \operatorname{pow}^{*}\left(\left\{{\overline{\alpha_{1}}}^{\left(r_{j}-1\right)}, \ldots,{\overline{\alpha_{\ell}}}^{\left(r_{j}-1\right)}\right\}\right),
$$

whereas $\bigcup_{\beta \in T(A)} \bar{\beta}^{\left(r_{j+1}-1\right)} \nsupseteq$ pow $^{*}\left(\left\{{\overline{\alpha_{1}}}^{\left(r_{j+1}-1\right)}, \ldots,{\overline{\alpha_{\ell}}}^{\left(r_{j+1}-1\right)}\right\}\right)$. Therefore, we deduce that $\bigcup_{i=1}^{l}{\overline{\alpha_{i}}}^{\left(r_{j}-1\right)} \subset \bigcup_{i=1}^{l}{\overline{\alpha_{i}}}^{\left(r_{j+1}-1\right)}, j=1,2,3, \ldots$ But $\left|\bigcup_{i=1}^{l}{\overline{\alpha_{i}}}^{\left(r_{1}-1\right)}\right|$ $\geq \ell$ and $\left|\bigcup_{i=1}^{\ell} \frac{\bar{\alpha}_{i}}{(r,-1)}\right| \leq(\rho-1) \ell$. Hence, if we denote by $N(A)$ the number of all the calls $C_{r}$, Distribute $\left(\alpha_{1}, \ldots, \alpha_{\ell}\right)$, made from the Propagation Phase and such that $\left|\bar{\alpha}_{i}^{(r,-1)}\right|<\rho$ for all $i=1, \ldots, \ell$, we have

$$
N(A) \leq(\rho-2)|A|+1 .
$$

Easy calculations prove

$$
\begin{aligned}
& \sum_{\substack{A \subseteq n \backslash\left\{\pi^{\pi v}\right\} \\
\wedge A \neq 0}} N(A) \\
= & \left(2^{n-1}-1\right)+(\rho-2) \sum_{i=1}^{n-1}\left(\begin{array}{c}
n-1 \\
i
\end{array}\right) i \\
= & (\rho-2)(n-1) 2^{n-2}+2^{n-1}-1 .
\end{aligned}
$$

Furthermore, it is easy to see that the empty node is processed just once, and that by Lemma 3.13(b)-(c) each nonempty P-node can be processed at most two times more by procedure Distribute. Therefore, the sequence of calls $C_{1}, C_{2}, C_{3}, \ldots$ in $K$ is finite and if we denote by $\xi$ its length the following inequality holds

$$
\xi \leq(\rho-2)(n-1) 2^{n-2}+3 \cdot 2^{n-1}-2,
$$

proving the first half of the Termination Lemma.

Next we will prove the slightly stronger fact that the computation $K$ necessarily terminates, i.e. reaches a point in which every place is blocked. This will be established by first proving that if this is false, the set $\bigcup_{\substack{\pi \in \mathbb{\pi} \\ \wedge \bar{\pi} \mid<\beta}}\left(\sigma^{\pi} \backslash f[\bar{\pi}]\right)$ must be nonempty, and then obtaining a contradiction from this fact.

Remark. As in Section 4, for simplicity we will often write $\bar{\pi}$ in place of $\bar{\pi}^{(\xi)}$, for all $\pi \in \Pi$, and $f$ in place of $f^{(\xi)}$, where $\xi$ is the length of the sequence of calls $C_{1}, C_{2}, C_{3}, \ldots$ in $\mathrm{K}$. 
Suppose therefore that $K^{*}$ does not terminate, i.e. that after the last call $C_{\xi}$ to the procedure Distribute, $K$ will remain permanently in a state $\Sigma$ in which there are unblocked places. It is easy to see by examining the I.V.A.A. code that this implies that the following two statements must be true.

(S.1) Let $\vartheta_{0}$ be the minimum unblocked place in state $\Sigma$. Then (the blocking phase never makes any additional node blocked, i.e.) either

(S.1.1) $\vartheta_{0}$ is trapped and $\left|\overline{\vartheta_{0}}\right| \neq\left|\sigma^{\nu_{0}}\right|$; or

(S.1.2) $\vartheta_{0}$ is nontrapped, $\left|\overline{\vartheta_{0}}\right| \geq \rho$ and for some P-node $\left\{\alpha_{1}, \ldots, \alpha_{\ell}\right\}$ having $\vartheta_{0}$ among its targets, and such that $\left|\overline{\alpha_{j}}\right|<\rho$ for all $j=1, \ldots, \ell$, we have

$$
\left|\operatorname{pow}^{*}\left(\left\{\overline{\alpha_{1}}, \ldots \overline{\alpha_{\ell}}\right\}\right) \cap \overline{\vartheta_{0}}\right| \neq\left|\operatorname{pow}^{*}\left(\left\{\sigma^{\alpha_{1}}, \ldots, \sigma^{\alpha_{\ell}}\right\}\right) \cap \sigma^{\vartheta_{0}}\right| ;
$$

or

(S.1.3) $\vartheta_{0}$ is nontrapped and $\left|\overline{\vartheta_{0}}\right|<\rho$.

(S.2) (No call to Distribute is made on behalf of any unblocked node, i.e.) for every unblocked $P$-node $\left\{\alpha_{1}, \ldots, \alpha_{\ell}\right\}$, either

(S.2.1) $\bar{\alpha}_{j_{0}}=\emptyset$, for some $j_{0} \in\{1, \ldots, \ell\}$; or

(S.2.2) $0<\left|\overline{a_{j}}\right|<\rho$, for all $j=1, \ldots, \ell$, and

$$
\operatorname{pow}^{*}\left(\left\{\overline{\alpha_{1}}, \ldots, \overline{\alpha_{\ell}}\right\}\right) \subseteq \bigcup_{\gamma \in T\left(\left\{\alpha_{1}, \ldots, \alpha_{\ell}\right\}\right)} \bar{\gamma} .
$$

To begin with, we establish the following lemma.

LEMMA 5.1 Assume that the foregoing statements (S.1) and (S.2) holds, and let $A=\left\{\alpha_{1}, \ldots, \alpha_{\ell}\right\}$ be a $P$-node such that $\left|\bar{\alpha}_{i}\right|<\rho$, for all $i=1, \ldots, \ell$. Then

$$
\operatorname{pow}^{*}\left(\bar{\alpha}_{1}, \ldots, \bar{\alpha}_{\ell}\right) \subseteq\left(\bigcup_{\beta \in T(A)} \bar{\beta}\right) \cap \operatorname{dom}(f) \text {. }
$$

Proof. In view of Assertion (E.7), it is enough to show that pow $\left(\overline{\alpha_{1}}, \ldots\right.$, $\left.\overline{\alpha_{\ell}}\right) \subseteq \bigcup_{\beta \in T(A)} \bar{\beta}$. If the P-node $\left\{\alpha_{1}, \ldots, \alpha_{\ell}\right\}$ is blocked, let $C_{r_{0}}$ be the last call Distribute $\left(\alpha_{1}, \ldots, \alpha_{\ell}\right)$. Then

$$
\begin{aligned}
& \operatorname{pow}^{*}\left(\left\{\overline{\alpha_{1}}, \ldots, \overline{\alpha_{\ell}}\right\}\right) \\
= & \operatorname{pow}^{*}\left(\left\{{\overline{\alpha_{1}}}^{\left(r_{0}-1\right)}, \ldots,{\overline{\alpha_{\ell}}}^{\left(\tau_{0}-1\right)}\right\}\right) \\
\subseteq & \bigcup_{\beta \in T(A)} \bar{\beta}^{\left(r_{0}\right)} \subseteq \bigcup_{\beta \in T(A)} \bar{\beta} .
\end{aligned}
$$


On the other hand, if the P-node is unblocked, our conclusion follows from statement (S.2), and in any case the lemma holds.

Next we prove the following lemma, from which it will by easy to deduce that $\bigcup_{\substack{\pi \in \Pi \\ \wedge|\bar{\pi}|<\rho}}\left(\sigma^{\pi} \backslash f[\bar{\pi}]\right) \neq \emptyset$.

LEMMA 5.2 The hypothesis that statements (S.1) and (S.2) hold implies that in state $\Sigma$ there is some place $\gamma$ of $\phi$ such that

$$
|\bar{\gamma}|<\min \left(\rho,\left|\sigma^{\gamma}\right|\right) \text {. }
$$

Proof. We distinguish three cases, according to whether (S.1.1), or (S.1.2), or (S.1.3) holds.

Case: (S.1.1) holds. Suppose that $\vartheta_{0}$ is a trapped place and that $\left|\overline{\vartheta_{0}}\right| \neq\left|\sigma^{\vartheta_{0}}\right|$. Therefore it follows from Assertion (E.5) that $\left|\overline{\vartheta_{0}}\right|<\mid \sigma^{\vartheta_{0}}<\rho$, which clearly yields $\left|\overline{\vartheta_{0}}\right|<\min \left(\rho,\left|\sigma^{\vartheta_{0}}\right|\right)$.

Case: (S.1.2) holds. Next assume that $\vartheta_{0}$ is nontrapped but for some $\mathrm{P}$-node $\left\{\alpha_{1}, \ldots, \alpha_{\ell}\right\}$ having $\vartheta_{0}$ as a target, and such that $\left|\overline{\alpha_{i}}\right|<\rho$ for all $i=1, \ldots, \ell$, we have

$$
\left|\operatorname{pow}^{*}\left(\left\{\overline{\alpha_{1}}, \ldots, \overline{\alpha_{\ell}}\right\}\right) \cap \overline{\vartheta_{0}}\right| \neq\left|p o w^{*}\left(\left\{\sigma^{a_{1}}, \ldots, \sigma^{\alpha_{\ell}}\right\}\right) \cap \sigma^{\vartheta_{0}}\right|
$$

The preceding lemma implies that $\operatorname{pow}^{*}\left(\left\{\overline{\alpha_{1}}, \ldots, \overline{\alpha_{\ell}}\right\}\right) \subseteq \operatorname{dom}(f)$. But Lemmas 2.7 and 5.1 yield

$$
\begin{aligned}
& f\left[p o w^{*}\left(\left\{\overline{\alpha_{1}}, \ldots, \overline{\alpha_{\ell}}\right\}\right) \cap \overline{\vartheta_{0}}\right] \\
= & \operatorname{pow}^{*}\left(\left\{f\left[\overline{\alpha_{1}}\right], \ldots, f\left[\overline{\alpha_{\ell}}\right]\right\}\right) \cap f\left[\overline{\vartheta_{0}}\right] \\
\subseteq & \operatorname{pow}^{*}\left(\left\{\sigma^{\alpha_{1}}, \ldots, \sigma^{\alpha_{\ell}}\right\}\right) \cap \sigma^{\vartheta_{0}} .
\end{aligned}
$$

Therefore, by the injectivity of $f$,

$$
\mid \text { pow }^{*}\left(\left\{\overline{\alpha_{1}}, \ldots, \overline{\alpha_{\ell}}\right\}\right) \cap \overline{\vartheta_{0}}|<| p_{0} w^{*}\left(\left\{\sigma^{\alpha_{1}}, \ldots, \sigma^{\alpha_{\ell}}\right\}\right) \cap \sigma^{\vartheta_{0}} \mid .
$$

Next we show that

$$
\operatorname{pow}^{*}\left(\left\{f\left[\overline{\alpha_{1}}\right], \ldots, f\left[\overline{\alpha_{\ell}}\right]\right\}\right) \cap f\left[\overline{\vartheta_{0}}\right]=\operatorname{pow}^{*}\left(\left\{f\left[\overline{\alpha_{1}}\right], \ldots, f\left[\overline{\alpha_{\ell}}\right]\right\}\right) \cap \sigma^{\vartheta_{0}} .
$$

Let $t \in \operatorname{pow}^{*}\left(\left\{f\left[\overline{\alpha_{1}}\right], \ldots, f\left[\overline{\alpha_{\ell}}\right]\right\}\right) \cap \sigma^{\vartheta_{0}}=f\left[p o w^{*}\left(\left\{\overline{\alpha_{1}}, \ldots, \overline{\alpha_{\ell}}\right\}\right)\right] \cap \sigma^{\vartheta_{0}}$. Then by Assertion (E.8), $t=f(u)$, for some $u \in$ pow $^{*}\left(\left\{\overline{\alpha_{1}}, \ldots, \overline{\alpha_{\ell}}\right\}\right) \cap \overline{\vartheta_{0}}$, which in particular proves $t=f(u) \in f\left[\bar{\vartheta}_{0}\right]$. Thus

$$
\operatorname{pow}^{*}\left(\left\{f\left[\overline{\alpha_{1}}\right], \ldots, f\left[\overline{\alpha_{\ell}}\right]\right\}\right) \cap \sigma^{\vartheta_{0}} \subseteq \operatorname{pow}^{*}\left(\left\{f\left[\overline{\alpha_{1}}\right], \ldots, f\left[\overline{\alpha_{\ell}}\right]\right\}\right) \cap f\left[\overline{\vartheta_{0}}\right] .
$$


The converse inclusion is an immediate consequence of Assertion (E.3), and therefore the equality follows.

This in turn implies

$$
\left|\operatorname{pow}^{*}\left(\left\{f\left[\overline{\alpha_{1}}\right], \ldots, f\left[\overline{\alpha_{\ell}}\right]\right\}\right) \cap \sigma^{\vartheta_{0}}\right|<\left|p o w^{*}\left(\left\{\sigma^{\alpha_{1}}, \ldots, \sigma^{\alpha_{\ell}}\right\}\right) \cap \sigma^{\vartheta_{0}}\right|,
$$

and thus, by Assertion (E.3), $f\left[\overline{\alpha_{j_{0}}}\right] \subset \sigma^{a, 0}$, for some $j_{0} \in\{1 \ldots, \ell\}$. Therefore $\left|\overline{\alpha_{j_{0}}}\right|<\min \left(\rho,\left|\sigma^{\alpha_{20}}\right|\right)$, proving our lemma, at least in the case in which (S.1.2) holds.

Case: (S.1.3) holds. Finally suppose that $\vartheta_{0}$ is nontrapped and that $\left|\overline{\vartheta_{0}}\right|<$ $\rho$. Then $\left|\overline{\vartheta_{0}}\right|<\rho \leq\left|\sigma^{\vartheta_{0}}\right|$, yielding $\left|\overline{\vartheta_{0}}\right|<\min \left(\rho,\left|\sigma^{\vartheta_{0}}\right|\right)$ and completing the proof of the lemma in all possible cases.

COROLLARY 5.3 If statements (S.1) and (S.2) hold, then

$$
\bigcup_{\substack{\pi \in \pi \\ \wedge|\bar{\pi}|<\infty}}\left(\sigma^{\pi} \backslash f[\bar{\pi}]\right) \neq 0
$$

Proof. The preceding lemma implies that there exists a place $\gamma$ such that $|\bar{\gamma}|<\min \left(\rho,\left|\sigma^{\gamma}\right|\right)$. Therefore $\emptyset \neq \sigma^{\gamma} \backslash f[\bar{\gamma}] \subseteq \bigcup_{\substack{\pi \in \pi \\ \wedge|\overline{\mid}|<\infty}}\left(\sigma^{\pi} \backslash f\left[\bar{x}^{*}\right)\right.$ and the corollary follows.

Now we are ready to show how to derive a contradiction from the fact that $\bigcup_{\substack{\pi \in \mathbb{R} \\ \wedge \bar{\pi} \mid<\infty}}\left(\sigma^{\pi} \backslash f[\bar{\pi}]\right) \neq \emptyset$

To this end let $t_{0} \in \sigma^{\pi_{0}} \backslash f\left[\overline{\pi_{0}}\right]$ be an element of minimal rank in $\bigcup_{\substack{\pi \in \Pi \\ \wedge|\bar{\pi}|<\rho}}\left(\sigma^{\pi} \backslash f[\bar{\pi}]\right)$, where $\left|\overline{\pi_{0}}\right|<\rho$.

From Lemma 3.6(c) it follows that $t_{0} \in \sigma^{\pi_{0}} \cap \operatorname{npow}^{*}\left(\left\{\sigma^{\alpha_{1}}, \ldots, \sigma^{\alpha_{\ell}}\right\}\right)$, for some P-node $A=\left\{\alpha_{1}, \ldots, \alpha_{\ell}\right\}$ having $\pi_{0}$ as a target.

There are only three possibilities: either $\overline{\alpha_{i_{0}}}=\emptyset$ for some $i_{0} \in\{1, \ldots, \ell\}$, or $0<\left|\overline{\alpha_{i}}\right|<\rho$ for all $i=1, \ldots, \ell$, or $\overline{\alpha_{i}} \neq \emptyset$ for all $i=1, \ldots, \ell$ and $\left|\overline{\alpha_{j_{0}}}\right| \geq \rho$ for some $j_{0} \in\{1, \ldots, \ell\}$. Below we will show that all cases lead to a contradiction, thereby proving that at the end of the computation $K$ all places must be blocked.

Case: $\overline{\alpha_{i_{0}}}=\emptyset$ for some $i_{0} \in\{1, \ldots, \ell\}$. Since $t_{0} \cap \sigma^{\alpha_{i_{0}}} \neq \emptyset$, for every $u_{0} \in$ $t_{0} \cap \sigma^{\alpha_{i_{0}}}$ we have $\operatorname{rank}\left(u_{0}\right)<\operatorname{rank}\left(t_{0}\right)$ and $u_{0} \in \sigma^{\alpha_{i_{0}}} \backslash f\left[\overline{\alpha_{i_{0}}}\right] \subseteq \bigcup_{\substack{\pi \in \square \\ \wedge|\bar{\pi}|<p}}\left(\sigma^{\pi} \backslash f[\bar{\pi}]\right)$, contradicting the minimality of $\operatorname{rank}\left(t_{0}\right)$ and ruling out this first case. 
Case: $0<\left|\overline{\alpha_{i}}\right|<\rho$ for all $i=1, \ldots, \ell$. As proved in Lemma 5.1 , in this case we have $\operatorname{pow}^{*}\left(\left\{\overline{\alpha_{1}}, \ldots, \overline{\alpha_{\ell}}\right\}\right) \subseteq\left(\bigcup_{\beta \in T(A)} \bar{\beta}\right) \cap \operatorname{dom}(f)$ and therefore by Lenmas 2.7 and 5.1

$$
f\left[p o w^{*}\left(\left\{\overline{\alpha_{1}}, \ldots \overline{\alpha_{\ell}}\right\}\right)\right]=\operatorname{pow}^{*}\left(\left\{f\left[\overline{\alpha_{1}}\right], \ldots, f\left[\overline{\alpha_{\ell}}\right]\right\}\right) \subseteq \operatorname{pow}^{*}\left(\left\{\sigma^{\alpha_{1}}, \ldots, \sigma^{\alpha_{\ell}}\right\}\right) .
$$

Thus, since

$$
t_{0} \in \operatorname{pow}^{*}\left(\left\{\sigma^{\alpha_{1}}, \ldots, \sigma^{\alpha_{\ell}}\right\}\right) \backslash \operatorname{pow}^{*}\left(\left\{f\left[\overline{\alpha_{1}}\right], \ldots, f\left[\overline{\alpha_{\ell}}\right]\right\}\right)
$$

it follows that for some place $\alpha_{i_{0}}$ there must exist an element $u_{0}$ such that $u_{0} \in t_{0} \cap \sigma^{\alpha_{10}} \backslash f\left[\bar{\alpha}_{i_{0}}\right]$. But then $\operatorname{rank}\left(u_{0}\right)<\operatorname{rank}\left(t_{0}\right)$ and $u_{0} \in \bigcup_{\substack{\pi \in \mathbb{\pi} \\ \wedge|\bar{\pi}|<\rho}}\left(\sigma^{\pi} \backslash f[\bar{\pi}]\right)$, which shows that the present case is inconsistent.

Finally we prove that even the last case is contradictory.

Case: $\bar{\alpha}_{i} \neq \emptyset$ for all $i=1, \ldots, \ell$ and $\left|\bar{\alpha}_{i_{0}}\right| \geq \rho$ for some $j_{0} \in\{1, \ldots, \ell\}$. In this case, statement ( $S .2)$ yields that the $\mathrm{P}$-node $\left\{\alpha_{1}, \ldots, \alpha_{\ell}\right\}$ is marked either visited or blocked. In any event, as $\left|\bar{\pi}_{0}\right|<\rho$ it follows that the place $\pi_{0}$ must be trapped. Thus by Assertion $D$ we have

$$
\left|\sigma^{\pi_{0}} \cap p o w^{*}\left(\left\{\sigma^{\alpha_{1}}, \ldots, \sigma^{\alpha_{l}}\right\}\right) \backslash \sigma^{A}\right|=\left|\bar{\pi} \cap p o w^{*}\left(\left\{\overline{\alpha_{1}}, \ldots, \overline{\alpha_{\ell}}\right\}\right)\right| .
$$

where

$$
\sigma^{A}= \begin{cases}\emptyset & \text { if } A \text { is blocked } \\ \left\{\sigma^{\alpha_{1}} \cup \ldots \cup \sigma^{\alpha_{2}}\right\} & \text { otherwise }\end{cases}
$$

Since $\sigma^{\pi_{0}} \cap$ pow $\left(\left\{\sigma^{\alpha_{1}}, \ldots, \sigma^{\alpha_{L}}\right\}\right) \backslash \sigma^{A} \subseteq$ range $(f)$, whereas by Assertion (E.3) $t_{0} \in \sigma^{\pi_{0}} \cap \operatorname{pow}^{*}\left(\left\{\sigma^{\alpha_{1}}, \ldots \sigma^{\alpha_{\ell}}\right\}\right) \backslash$ range $(f)$, the only possibility is that the Pnode $A$ is unblocked and $t_{0}=\sigma^{\alpha_{1}} \cup \ldots \cup \sigma^{\alpha_{\ell}}$. In particular some $\alpha_{k_{0}} \in A$ must be unblocked. Thus $\vartheta_{0} \leq \alpha_{k_{0}}$ and consequently $\operatorname{rank}\left(\sigma^{\vartheta_{0}}\right) \leq \operatorname{rank}\left(\sigma^{\alpha_{k_{0}}}\right)$ (cf. Definition 3.8).

If $\left|\overline{\vartheta_{0}}\right|<\rho$, then Assertion (E.4) and statement (S.1) yield $\theta \neq \sigma^{\vartheta_{0}} \backslash f\left[\overline{\vartheta_{0}}\right] \subseteq$ $\bigcup_{x \in \mathbb{I}}\left(\sigma^{\pi} \backslash f[\bar{\pi}]\right)$, and therefore for every $t_{1} \in \sigma^{\vartheta_{0}} \backslash f\left[\overline{\vartheta_{0}}\right]$ we have $\underset{\substack{x \in \mathbb{Z} \\ \wedge|\bar{x}|<\rho}}{ }$

$$
\begin{aligned}
\operatorname{rank}\left(t_{1}\right) & <\operatorname{rank}\left(\sigma^{\vartheta_{0}}\right) \leq \operatorname{rank}\left(\sigma^{\alpha_{k_{0}}}\right) \\
& \leq \operatorname{rank}\left(\sigma^{\alpha_{1}} \cup \ldots \cup \sigma^{\alpha_{\ell}}\right)=\operatorname{rank}\left(t_{0}\right) .
\end{aligned}
$$

This contradicts the minimality of $\operatorname{rank}\left(t_{0}\right)$ and rules out the possibility that $\left|\overline{\vartheta_{0}}\right|<\rho$. 
On the other hand, if $\left|\overline{\vartheta_{0}}\right| \geq \rho$, by statement (S.1) we deduce that for some $\mathrm{P}$-node $\left\{\beta_{1} \ldots, \beta_{k}\right\}$ having $\vartheta_{0}$ among its targets, and such that $\left|\overline{\beta_{j}}\right|<\rho$ for all $j=1, \ldots, k$, we have

$$
\left|\operatorname{pow}^{*}\left(\left\{\overline{\beta_{1}}, \ldots, \overline{\beta_{k}}\right\}\right) \cap \overline{\vartheta_{0}}\right| \neq\left|\operatorname{pow}^{*}\left(\left\{\sigma^{\beta_{1}}, \ldots \sigma^{\beta_{k}}\right\}\right) \cap \sigma^{\vartheta_{0}}\right| .
$$

This implies

$$
\operatorname{pow}^{*}\left(\left\{\sigma^{\beta_{1}}, \ldots, \sigma^{\beta_{k}}\right\}\right) \cap \sigma^{\vartheta_{0}} \backslash f\left(p o w^{*}\left(\left\{\overline{\beta_{1}}, \ldots, \overline{\beta_{k}}\right\}\right) \cap \overline{\vartheta_{0}}\right] \neq \emptyset
$$

which by Assertion (E.4) in turn gives

$$
\operatorname{pow}^{*}\left(\left\{\sigma^{\mathcal{G}_{1}}, \ldots, \sigma^{\beta_{k}}\right\}\right) \cap \sigma^{\vartheta_{0}} \backslash f\left[p o w^{*}\left(\left\{\overline{\beta_{1}}, \ldots, \overline{\beta_{k}}\right\}\right)\right] \neq \emptyset,
$$

i.e.,

$$
\operatorname{pow}^{*}\left(\left\{\sigma^{\beta_{1}}, \ldots, \sigma^{\beta_{k}}\right\}\right) \cap \sigma^{\vartheta_{0}} \backslash \operatorname{pow}^{*}\left(\left\{f\left[\overline{\beta_{1}}\right], \ldots, f\left[\overline{\beta_{k}}\right]\right\}\right) \neq 0 .
$$

Let $u \in \operatorname{pow}^{*}\left(\left\{\sigma^{\beta_{1}}, \ldots, \sigma^{\beta_{k}}\right\}\right) \cap \sigma^{\vartheta_{0}} \backslash p \operatorname{pow}\left(\left\{f\left[\overline{\beta_{1}}\right], \ldots, f\left[\overline{\beta_{k}}\right]\right\}\right)$. For some $i_{0} \in$ $\{1, \ldots, k\}, u \cap \sigma^{\beta_{i_{0}}} \subseteq f\left[\overline{\beta_{i_{0}}}\right]$. Hence, for each $u^{\prime} \in u \cap \sigma^{\beta_{i_{0}}} \backslash f\left[\overline{\beta_{i_{0}}}\right]$,

$$
u^{\prime} \in \bigcup_{\substack{\pi \in \mathbb{M} \\ \wedge|\bar{x}|<\phi}}\left(\sigma^{\pi} \backslash f[\bar{\pi}]\right)
$$

and

$$
\begin{aligned}
\operatorname{rank}\left(u^{\prime}\right)<\operatorname{rank}(u)<\operatorname{rank}\left(\sigma^{\vartheta_{0}}\right) & \leq \operatorname{rank}\left(\sigma^{\alpha_{k_{0}}}\right) \\
& \leq \operatorname{rank}\left(\sigma^{\alpha_{1}} \cup \ldots \cup \sigma^{\alpha_{\ell}}\right)=\operatorname{rank}\left(t_{0}\right),
\end{aligned}
$$

contradicting again the minimality of $\operatorname{rank}\left(t_{0}\right)$.

Having shown that a contradiction is derived even in this last case, it follows that our initial assumption (i.e. that there are unblocked places after the last call $C_{\xi}$ ) is false.

This concludes the proof of the Termination Lemma.

\section{Acknowledgments.}

The author is grateful to Jacob T. Schwartz for his invaluable assistance, and to E. Omodeo and A. Ferro for helpful suggestions and discussions.

The author also acknowledge partial support by ENI and ENIDATA within the AXL project, by the C.N.R. of Italy and by the U.S. NSF grant \# DCR84-01633. 


\section{References}

[Beh] Behmann,H., Beiträge zur algebra der logik insbesondere zum entscheidnigsproblem, Math. Annalen 8b, 1922, pp.163-220.

[Ble1] Bledsoe,W.W., Splitting and reduction heuristics in automatic theorem proving, Art. Int. 2, 1971, pp.55-77.

[Ble2] Bledsoe,W.W., Nonresolution theorem proving, Art. Int. 9, 1977.

[Ble3] Bledsoe,W.W., The UT interactive prover, Tech. Report ATP-17B, Univ. of Texas at Austin (1983).

[Bre] Breban,M., Ph. D. Dissertation, Courant Inst., New York Univ., 1981.

[BrF] Breban, M., and Ferro,A., Decision procedures for elementary sublanguages of set theory. III. Formulas involving a limited number of occurrences of the powerset and general union operators, Adv. in Appl. Math., 5, 1984.

[BFOS] Breban,M., Ferro,A., Omodeo,E.G., and Schwartz,J.T., Decision procedures for elementary sublanguages of set theory. II. Formulae involving restricted quantifiers together with ordinal, integer, map and domain notions. Comm. Pure Appl. Math. XXXIV, 2, 1981.

[BoM] Boyer,R.S. and Moore,J.S., Computational Logic, Academic Press, New York, 1979.

[Can] Cantone,D., A decision procedure for a class of unquantified formulae of set theory involving the powerset and singleton operators, $\mathrm{Ph} . \mathrm{D}$. Thesis, Courant Institute, New York Univ. (1987).

[CFMS] Cantone,D., Ferro,A., Micale,B., and Sorace,G., Decision procedures for elcmentary sublanguages of set theory. IV. Formulae involving a rank operator or one occurrence of the set operator $\Sigma(x)=\{(y) \mid y \in x\}$, Comm. Pure Appl. Math. XXXVII, 37-77 (1987).

[CFO] Cantone,D., Ferro,A., and Omodeo,E.G., Decision procedures for elementary sublanguages of set theory. VIII. A semidecision procedure for finite satisfiability of unquantified set-theoretic formulae, Comm. Pure Appl. Math. XLI 105-120 (1988). 
[CFOS] Cantone,D., Ferro,A., Omodeo,E., and Schwartz,J.T., Decision algorithms for some fragments of analysis and related areas, Comm. Pure Appl. Math. XL 281-300 (1987).

[CFS1] Cantone,D., Ferro,A., and Schwartz,J.T., Decision procedures for elementary sublanguages of set theory. V. Multilevel syllogistic extended by the general union operator. Jour. Comp. Syst. Sci., 34,1 (1987).

[CFS2] Cantone,D., Ferro,A., and Schwartz,J.T., Decision procedures for elementary sublanguages of set theory. VI. Multilevel syllogistic extended by the powerset operator. Comm. Pure Appl. Math. XXXVIII 549-571 (1985).

[CGO] Cantone,D., Ghelfo,S., and Omodeo,E.G., The automation of syllogistic. I. Syllogistic normal forms, Journ. Symb., Comp., to appear (1987).

[COP] Cantone,D., Omodeo,E.G., and Papoulias,A., The automation of syllogistic. II. An effective satisfiability test, Journ. Symbolic Comp., submitted (1987).

[Con] Constable,R.L., et al. Implementing mathematics with the NUPRL proof development system, Prentice Hall, Inc., Englewood Cliffs, New Jersey (1986).

[Fer1] Ferro,A., Decision procedures for some classes of unquantified set theoretic formulae, Ph. D. Dissertation, Courant Inst., New York Univ. (1981).

[Fer2] Ferro,A., A note on the decidability of MLS extended by the powerset operator, Comm. Pure Appl. Math., Vol. XXXVIII, 1985, pp.367-374.

[FeO Ferro,A., and Omodeo,E., Decision procedures for elementary sublanguages of set theory. VII. Validity in set theory when a choice operator is present, Comm. Pure Appl. Math., XL, 265-280 (1987).

[FOS1] Ferro,A., Omodeo,E., and Schwartz,J.T., Decision procedures for elementary sublanguages of set theory. I. Multilevel syllogistic and some extensions. Comm. Pure Appl. Math. 33, 1980.

[FOS2] Ferro,A., Omodeo,E., and Schwartz,J.T., Decision procedures for elementary fragments of set theory, Fifth Conf. on Automated Deduction, Les Arcs, France, Lect. Notes in Comp. Sci. 87, Springer-Verlag. 
[Gog1] Gogol,D., The $\forall_{n}-\exists$ completeness of Zermelo-Fraenkel set theory, Zeitschr. f. math. Logik und Grundlagen d. Math. 2, 1978, pp.289-290.

[Gog2] Gogol,D., Sentences with three quantifier are decidable, Fund. Math. CII, 1979, pp.1-8.

[Jec] Jech,T., Set Theory, Academic Press, New York, 1978.

[KeW] Ketonen,J., Weening,J., EKL - an interactive proof checker, Users' Ref. Manual, 40 pp, Stanford Univ., 1983.

[LuO] Lusk,E.L., and Overbeek,R.A., The automated reasoning system ITP, ANL-8427, Argonne Natl. Lab. (April 1984).

[NeO] Nelson,C.G., Oppen,D.C., Simplifier based on Efficient Decision Algorithms, Fifth, Ann. Symp. on Principles of Programming Languages (1978) 141-150.

[Omo] Omodeo,E.G., Decidability and proof procedures for set theory, Ph.D. Thesis, Courant Inst., New York Univ., 1984.

[PaP] Parlamento,F., Policriti,A., Decision procedures for elementary sublanguages of set theory. IX. Undecidability of set theoretic formulas involving restricted quantifiers, Comm. Pure App. Math. XLI 221-251 (1988).

[Pas] Pastre,D., Automatic Theorem Proving in Set Theory, Art. Int. 10 (1978) 1-27.

[Sch1] Schwartz,J.T., Instantiation and decision procedures for certain classes of quantified set theoretical formulae. Inst. for Comp. Appl. in Science and Engineering, NASA Langley Research Center, Hampton, VA, Report \# 7810, 1978.

[Sch2] Schwartz,J.T., A survey of program proof technology, New York Univ., Comp. Sci. Dept., Report \# 1, September 1978.

[Sla] Slagle,J.R., Automatic Theorem Proving with Built-In Theories Including Equality, Partial Ordering and Sets. J. ACM 19 (1972) 120-135.

[Tho] Thompson,D.H., ed., AFFIRM reference manual, USC Information Sciences Institute, 1979. 
[Vil] Ville,F., Decidabilité des formules existentielles en theorie des ensembles, C.R. Acad Sci., Paris, t. 272 Série A, 1971, pp. 513-519.

[Wey] Weyhrauch,R.W., FOL: a proof checker for first-order logic, Stanford Artificial Intelligence Lab. Memo AIM-253.1 (1977). 

NYU COMPSCI TR-373 C.2

Cantone, D

Decision procedures for

elementary sublanguages of set theory. $x$.

- NYU COMPSCI TR-373 c.2 Cantone, D

Decision procedures for elementary sublanguages of - set theory. $x$.

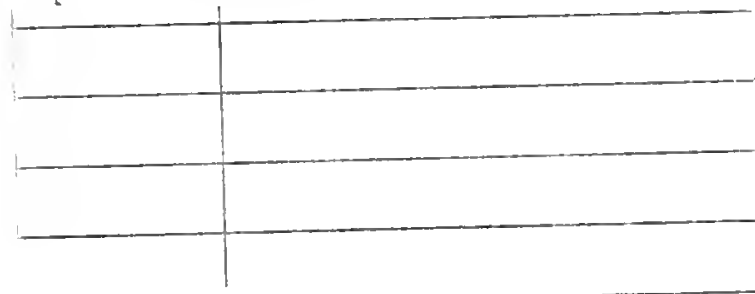

This bonk may be kept

\section{FOURTHENTIAYS}

A fine will he charged for each in the book is hept overtim

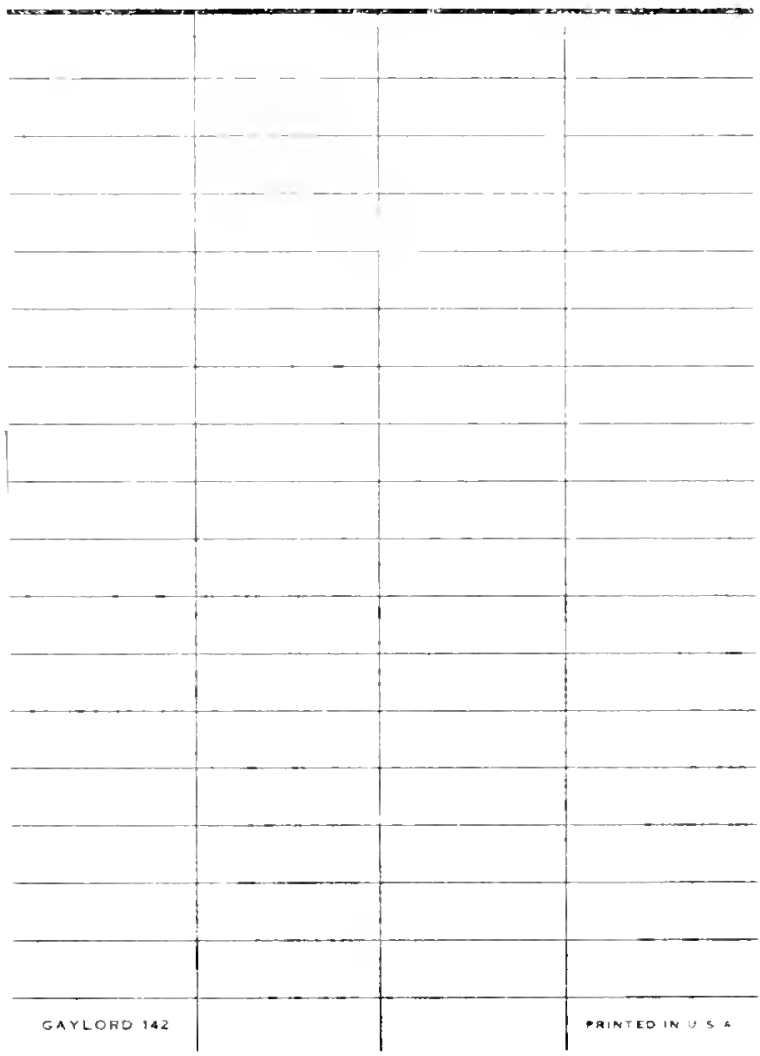


